\title{
Article \\ Ostrowski Type Inequalities Involving Harmonically Convex Functions and Applications
}

\author{
Nousheen Akhtar ${ }^{1,+}{ }^{+}$, Muhammad Uzair Awan ${ }^{1, \dagger}$, Muhammad Zakria Javed ${ }^{1, \dagger}$, Michael Th. Rassias ${ }^{2, *, t, \ddagger}$, \\ Marcela V. Mihai ${ }^{3,+}$, Muhammad Aslam Noor ${ }^{4,+}$ (D) and Khalida Inayat Noor ${ }^{4,+}$ (D) \\ 1 Department of Mathematics, Government College University, Faisalabad 38000, Pakistan; \\ nousheenakhtar86@gmail.com (N.A.); muawan@gcuf.edu.pk (M.U.A.); zakriajaved071@gmail.com (M.Z.J.) \\ 2 Institute of Mathematics, University of Zurich, 8057 Zurich, Switzerland \\ 3 Romanian Mathematical Society-Branch Bucharest, Academy Street no. 14, RO-010014 Bucharest, Romania; \\ marcelamihai58@yahoo.com \\ 4 Department of Mathematics, COMSATS University Islamabad, Islamabad 44000, Pakistan; \\ aslamnoor@comsats.edu.pk (M.A.N.); khalidainayat@comsats.edu.pk (K.I.N.) \\ * Correspondence: michail.rassias@math.uzh.ch \\ + These authors contributed equally to this work. \\ $\ddagger$ Current address: Romanian Mathematical Society-Branch Bucharest, Academy Street no. 14, RO-010014 \\ Bucharest, Romania.
}

check for updates

Citation: Akhtar, N.; Awan, M.U.; Javed, M.Z.; Rassias, M.T.; Mihai,

M.V.; Noor, M.A.; Noor, K.I. Ostrowski Type Inequalities Involving Harmonically Convex Functions and Applications. Symmetry 2021, 13, 201. https:// doi.org/10.3390/sym13020201

Academic Editor: Sun Young Cho Received: 22 December 2020 Accepted: 25 January 2021 Published: 27 January 2021

Publisher's Note: MDPI stays neutral with regard to jurisdictional clai$\mathrm{ms}$ in published maps and institutional affiliations.

Copyright: (C) 2021 by the authors. Licensee MDPI, Basel, Switzerland. This article is an open access article distributed under the terms and conditions of the Creative Commons Attribution (CC BY) license (https:// creativecommons.org/licenses/by/ $4.0 /)$.

\begin{abstract}
The main objective of this paper is to derive some new generalizations of Ostrowski type inequalities for the functions whose first derivatives absolute value are harmonically convex. We also discuss some special cases of the obtained results. In the last section, we present some applications of the obtained results.
\end{abstract}

Keywords: convex; harmonic; derivative; ostrowski; inequality

\section{Introduction and Preliminaries}

A function $\Lambda: I \subset \mathbb{R} \rightarrow \mathbb{R}$ is said to be convex, if

$$
\Lambda\left((1-\tau) a_{1}+t b\right) \leq(1-\tau) \Lambda\left(a_{1}\right)+\tau \Lambda\left(a_{2}\right), \quad \forall a_{1}, a_{2} \in I, \tau \in[0,1]
$$

Iscan [1] introduced the notion of harmonically convex functions as follows: A function $\Lambda: I \subset(0, \infty) \rightarrow \mathbb{R}$ is said to be harmonically convex, if

$$
\Lambda\left(\frac{a_{1} a_{2}}{\tau a_{1}+(1-\tau) a_{2}}\right) \leq(1-\tau) \Lambda\left(a_{1}\right)+\tau \Lambda\left(a_{2}\right), \quad \forall a_{1}, a_{2} \in I, \tau \in[0,1] .
$$

It is worth mentioning here that the harmonic property has played a significant role in different fields of pure and applied sciences. In [2], the authors have discussed the important role of the harmonic mean in Asian stock options. Interestingly, harmonic means are applied in electric circuit theory. More specifically, the total resistance of a set of parallel resistors is just half of the harmonic mean of the total resistors. For example, if $R_{1}$ and $R_{2}$ are the resistances of two parallel resistors, then the total resistance is computed by the formula:

$$
R_{T}=\frac{R_{1} R_{2}}{R_{1}+R_{2}}=\frac{1}{2} H\left(R_{1}, R_{2}\right),
$$

which is the half of the harmonic mean.

Noor [3] showed that the harmonic mean also plays a crucial role in the development of parallel algorithms for solving nonlinear problems. Several authors have used harmonic means and harmonic convex functions to propose some iterative methods for solving linear and nonlinear system of equations. 
Convexity played a vital role in the development of the theory of inequalities. Several inequalities constitute direct consequences of applications of convexity. In this regard, Hermite-Hadamard's inequality is one of the most extensively as well as intensively studied result. It provides us with an estimate of the (integral) mean value of a continuous convex function. It reads as:

Let $\Lambda: I=\left[a_{1}, a_{2}\right] \subset \mathbb{R} \rightarrow \mathbb{R}$ be a convex function, then

$$
\Lambda\left(\frac{a_{1}+a_{2}}{2}\right) \leq \frac{1}{a_{2}-a_{1}} \int_{a_{1}}^{a_{2}} \Lambda(x) \mathrm{d} x \leq \frac{\Lambda\left(a_{1}\right)+\Lambda\left(a_{2}\right)}{2} .
$$

Iscan [4] extended the classical version of Hermite-Hadamard's inequality using the harmonic convexity property of the function.

Let $\Lambda: I=\left[a_{1}, a_{2}\right] \subset(0, \infty) \rightarrow \mathbb{R}$ be a harmonically convex function, then

$$
\Lambda\left(\frac{2 a_{1} a_{2}}{a_{1}+a_{2}}\right) \leq \frac{a_{1} a_{2}}{a_{2}-a_{1}} \int_{a_{1}}^{a_{2}} \Lambda(x) \mathrm{d} x \leq \frac{\Lambda\left(a_{1}\right)+\Lambda\left(a_{2}\right)}{2} .
$$

An interesting problem related to Hermite-Hadamard's inequality is its precision. Note that the left Hermite-Hadamard inequality can be estimated by the following inequality:

$$
\left|\Lambda(x)-\frac{1}{a_{2}-a_{1}} \int_{a_{1}}^{a_{2}} \Lambda(x) \mathrm{d} x\right| \leq\left[\frac{1}{4}+\left(\frac{x-\frac{a_{1}+a_{2}}{2}}{a_{2}-a_{1}}\right)^{2}\right] M\left(a_{2}-a_{1}\right),
$$

where $M$ is the Lipschitz constant which is equal to $\sup \left\{\left|\frac{\Lambda(x)-\Lambda(y)}{x-y}\right| ; x \neq y\right\}$. This above inequality is known in the literature as Ostrowski's inequality.

Recently, several research articles have been written on different generalizations of Ostrowski's inequality using different techniques. For example, Alomari et al. [5] obtained Ostrowski type inequalities using the class of $s$-convex functions. Ardic et al. [6] obtained Ostrowski type inequalities using the class of GG-convex and $G A$-convex functions. Budak and Sarikaya [7] obtained generalized Ostrowski type inequalities for functions whose first derivatives' absolute values are convex. Budak and Sarikaya [8] also obtained some new weighted Ostrowski type inequalities for functions of two variables with bounded variation. Iscan [4] obtained some Ostrowski type inequalities using the class of harmonically sconvex functions. Khurshid et al. [9] obtained a conformable fractional version of Ostrowski type inequalities using preinvex functions. Koroglu [10] obtained some more generalized Ostrowski type inequalities using harmonically convex functions. Mohsin et al. [11] obtained new generalizations of Ostrowski type inequalities using harmonically $h$-convex functions. Set [12] obtained some generalized fractional refinements of Ostrowski type inequalities using the class of s-convex functions. Recently, Sun [13] obtained some more local fractional versions of Ostrowski type inequalities and discussed its applications. For more details on Ostrowski type inequalities and its applications, cf. [14].

The aim of this paper is to obtain some new generalizations of Ostrowski's inequality essentially utilising the harmonic convexity property of the functions. We first derive a new auxiliary result which will play a significant role in the development of these results. We also discuss some special cases that can be deduced from the main results of the paper. In the last section, we present some applications of the obtained results. We hope that the ideas and techniques presented within this paper will inspire interested readers.

\section{Main Results}

In this section, we discuss our main results. 
Let $L\left[a_{1}, a_{2}\right]$ be the space of Lebesgue integrable functions on the interval $\left[a_{1}, a_{2}\right]$. Our first result is an auxiliary lemma. This result will play a significant role in the development of the next results.

Lemma 1. Let $\Lambda:\left[a_{1}, a_{2}\right] \subset(0, \infty) \rightarrow \mathbb{R}$ be a differentiable mapping on $\left(a_{1}, a_{2}\right)$ with $a_{1}<a_{2}$. If $\Lambda^{\prime}$ belongs to $L\left[a_{1}, a_{2}\right]$, then, for all $\varrho \in(0,1)$ and for all $x \in\left[a_{1}, a_{2}\right]$, the following equality holds true:

$$
\begin{aligned}
& \int_{0}^{1} h(\tau, \varrho) \Lambda^{\prime}\left(\frac{a_{1} a_{2}}{\tau\left(\zeta a_{2}+(1-\varsigma) a_{1}\right)+(1-\tau)\left(\varsigma a_{1}+(1-\varsigma) a_{2}\right)}\right) \\
& \quad \times \frac{a_{1} a_{2}\left(a_{2}-a_{1}\right)(1-2 \varsigma)}{\left[\tau\left((1-\varsigma) a_{1}+\varsigma a_{2}\right)+(1-\tau)\left(\varsigma a_{1}+(1-\varsigma) a_{2}\right)\right]^{2}} \mathrm{~d} \tau \\
& =\varrho \frac{a_{1}\left(a_{2}-x\right) \Lambda\left(\frac{a_{1} a_{2}}{\zeta a_{1}+(1-\varsigma) a_{2}}\right)+a_{2}\left(x-a_{1}\right) \Lambda\left(\frac{a_{1} a_{2}}{(1-\zeta) a_{1}+\varsigma a_{2}}\right)}{x\left(a_{2}-a_{1}\right)} \\
& \quad+(1-\varrho) \Lambda\left(\frac{a_{1} a_{2}}{(1-\varsigma)\left(x\left(a_{1}+a_{2}\right)-a_{1} a_{2}\right)+\varsigma a_{1} a_{2}}\right)-\frac{a_{1}}{\left(a_{2}-a_{1}\right)(1-2 \zeta)} \int_{\frac{a_{1} a_{2}}{\zeta a_{1}+(1-\zeta) a_{2}}}^{\frac{a_{1} a_{2}}{(1-5) a_{1}+\zeta a_{2}}} \frac{\Lambda(s)}{s^{2}} \mathrm{~d} s \\
& =T(\Lambda, \varrho, \varsigma, x) .
\end{aligned}
$$

for $\varsigma \in[0,1] \backslash\left\{\frac{1}{2}\right\}$, where

$$
h(\tau, \varrho)=\left\{\begin{array}{rr}
\tau-\varrho \frac{a_{1}\left(a_{2}-x\right)}{x\left(a_{2}-a_{1}\right)}, & \tau \in\left[0, \frac{a_{1}\left(a_{2}-x\right)}{x\left(a_{2}-a_{1}\right)}\right], \\
\tau-1+\varrho \frac{a_{2}\left(x-a_{1}\right)}{x\left(a_{2}-a_{1}\right)}, & \tau \in\left(\frac{a_{1}\left(a_{2}-x\right)}{x\left(a_{2}-a_{1}\right)}, 1\right] .
\end{array}\right.
$$

Proof. For the sake of brevity, we write $A_{\tau}=\tau\left(\varsigma a_{2}+(1-\varsigma) a_{1}\right)+(1-\tau)\left(\varsigma a_{1}+(1-\varsigma) a_{2}\right)$. Let

$$
\begin{aligned}
I= & \int_{0}^{1} h(\tau, \varrho) \Lambda^{\prime}\left(\frac{a_{1} a_{2}}{A_{\tau}}\right) \frac{a_{1} a_{2}\left(a_{2}-a_{1}\right)(1-2 \varsigma)}{A_{\tau}^{2}} \mathrm{~d} \tau \\
= & \int_{0}^{\frac{a_{1}\left(a_{2}-x\right)}{x\left(a_{2}-a_{1}\right)}}\left[\tau-\varrho \frac{a_{1}\left(a_{2}-x\right)}{x\left(a_{2}-a_{1}\right)}\right] \Lambda^{\prime}\left(\frac{a_{1} a_{2}}{A_{\tau}}\right) \frac{a_{1} a_{2}\left(a_{2}-a_{1}\right)(1-2 \varsigma)}{A_{\tau}^{2}} \mathrm{~d} \tau \\
& +\int_{\frac{a_{1}\left(a_{2}-x\right)}{x\left(a_{2}-a_{1}\right)}}^{1}\left[\tau-1+\varrho \frac{a_{2}\left(x-a_{1}\right)}{x\left(a_{2}-a_{1}\right)}\right] \Lambda^{\prime}\left(\frac{a_{1} a_{2}}{A_{\tau}}\right) \frac{a_{1} a_{2}\left(a_{2}-a_{1}\right)(1-2 \varsigma)}{A_{\tau}^{2}} \mathrm{~d} \tau \\
= & I_{1}+I_{2} .
\end{aligned}
$$

Now, an integration by parts yields

$$
\begin{aligned}
I_{1}= & \int_{0}^{\frac{a_{1}\left(a_{2}-x\right)}{x\left(a_{2}-a_{1}\right)}}\left[\tau-\varrho \frac{a_{1}\left(a_{2}-x\right)}{x\left(a_{2}-a_{1}\right)}\right] \Lambda^{\prime}\left(\frac{a_{1} a_{2}}{A_{\tau}}\right) \frac{a_{1} a_{2}\left(a_{2}-a_{1}\right)(1-2 \varsigma)}{A_{\tau}^{2}} \mathrm{~d} \tau \\
= & (1-\varrho) \frac{a_{1}\left(a_{2}-x\right)}{x\left(a_{2}-a_{1}\right)} \Lambda\left(\frac{x a_{1} a_{2}}{(1-\varsigma)\left(x\left(a_{1}+a_{2}\right)-a_{1} a_{2}\right)+\varsigma a_{1} a_{2}}\right) \\
& +\frac{\rho a_{1}\left(a_{2}-x\right)}{x\left(a_{2}-a_{1}\right)} \Lambda\left(\frac{a_{1} a_{2}}{\varsigma a_{1}+(1-\varsigma) a_{2}}\right)-\int_{0}^{\frac{a_{1}\left(a_{2}-x\right)}{x\left(a_{2}-a_{1}\right)}} \Lambda\left(\frac{a_{1} a_{2}}{A_{\tau}}\right) \mathrm{d} \tau .
\end{aligned}
$$


Similarly,

$$
\begin{aligned}
I_{2}= & \int_{\frac{a_{1}\left(a_{2}-x\right)}{x\left(a_{2}-a_{1}\right)}}^{1}\left[\tau-1+\varrho \frac{a_{2}\left(x-a_{1}\right)}{x\left(a_{2}-a_{1}\right)}\right] \Lambda^{\prime}\left(\frac{a_{1} a_{2}}{A_{\tau}}\right) \frac{a_{1} a_{2}\left(a_{2}-a_{1}\right)(1-2 \varsigma)}{A_{\tau}^{2}} \mathrm{~d} \tau \\
= & \frac{\rho a_{2}\left(x-a_{1}\right)}{x\left(a_{2}-a_{1}\right)} \Lambda\left(\frac{a_{1} a_{2}}{(1-\varsigma) a_{1}+\varsigma a_{2}}\right) \\
& +(1-\varrho) \frac{a_{2}\left(x-a_{1}\right)}{x\left(a_{2}-a_{1}\right)} \Lambda\left(\frac{x a_{1} a_{2}}{(1-\varsigma)\left(x\left(a_{1}+a_{2}\right)-a_{1} a_{2}\right)+\varsigma a_{1} a_{2}}\right)-\int_{\frac{a_{1}\left(a_{2}-x\right)}{x\left(a_{2}-a_{1}\right)}}^{1} \Lambda\left(\frac{a_{1} a_{2}}{A_{\tau}}\right) \mathrm{d} \tau .
\end{aligned}
$$

Summing $I_{1}$ and $I_{2}$, we have

$$
\begin{aligned}
I= & \varrho \frac{a_{1}\left(a_{2}-x\right) \Lambda\left(\frac{a_{1} a_{2}}{\varsigma a_{1}+(1-\varsigma) a_{2}}\right)+a_{2}\left(x-a_{1}\right) \Lambda\left(\frac{a_{1} a_{2}}{(1-\zeta) a_{1}+\zeta a_{2}}\right)}{x\left(a_{2}-a_{1}\right)} \\
& +(1-\varrho) \Lambda\left(\frac{x a_{1} a_{2}}{(1-\varsigma)\left(x\left(a_{1}+a_{2}\right)-a_{1} a_{2}\right)+\varsigma a_{1} a_{2}}\right)-\int_{0}^{1} \Lambda\left(\frac{a_{1} a_{2}}{A_{\tau}}\right) \mathrm{d} \tau .
\end{aligned}
$$

This implies

$$
\begin{aligned}
I= & \varrho \frac{a_{1}\left(a_{2}-x\right) \Lambda\left(\frac{a_{1} a_{2}}{\zeta a_{1}+(1-\zeta) a_{2}}\right)+a_{2}\left(x-a_{1}\right) \Lambda\left(\frac{a_{1} a_{2}}{(1-\zeta) a_{1}+\zeta a_{2}}\right)}{x\left(a_{2}-a_{1}\right)} \\
& +(1-\varrho) \Lambda\left(\frac{x a_{1} a_{2}}{(1-\varsigma)\left(x\left(a_{1}+a_{2}\right)-a_{1} a_{2}\right)+\varsigma a_{1} a_{2}}\right)-\frac{a_{1} a_{2}}{\left(a_{2}-a_{1}\right)(1-2 \varsigma)} \int_{\frac{a_{1} a_{2}}{\zeta a_{1}+(1-\zeta) a_{2}}}^{\frac{a_{1} a_{2}}{\left(1-\zeta a_{1}\right)+\zeta a_{2}}} \frac{\Lambda(s)}{s^{2}} \mathrm{~d} s .
\end{aligned}
$$

This completes the proof.

We now discuss some special cases of Lemma 1. To the best of our knowledge, these special cases are also new in the literature.

I. If we set $\varsigma=1$ in Lemma 1 , then the following equality holds:

$$
\begin{aligned}
& \int_{0}^{1} h(\tau, \varrho) \Lambda^{\prime}\left(\frac{a_{1} a_{2}}{t b+(1-\tau) a_{1}}\right) \frac{a_{1} a_{2}\left(a_{1}-a_{2}\right)}{\left(t b+(1-\tau) a_{1}\right)^{2}} \mathrm{~d} \tau \\
& =\varrho \frac{a_{1}\left(a_{2}-x\right) \Lambda\left(a_{2}\right)+a_{2}\left(x-a_{1}\right) \Lambda\left(a_{1}\right)}{x\left(a_{2}-a_{1}\right)}+(1-\varrho) \Lambda(x)-\frac{a_{1} a_{2}}{\left(a_{2}-a_{1}\right)} \int_{a_{1}}^{a_{2}} \frac{\Lambda(s)}{s^{2}} \mathrm{~d} s .
\end{aligned}
$$

where

$$
h(\tau, \varrho)=\left\{\begin{array}{rr}
\tau-\varrho \frac{a_{1}\left(a_{2}-x\right)}{x\left(a_{2}-a_{1}\right)}, & \tau \in\left[0, \frac{a_{1}\left(a_{2}-x\right)}{x\left(a_{2}-a_{1}\right)}\right], \\
\tau-1+\varrho \frac{a_{2}\left(x-a_{1}\right)}{x\left(a_{2}-a_{1}\right)}, & \tau \in\left(\frac{a_{1}\left(a_{2}-x\right)}{x\left(a_{2}-a_{1}\right)}, 1\right] .
\end{array}\right.
$$

In addition, if we set $\varrho=0$ in (3), then the following equality holds:

$$
\begin{aligned}
& \int_{0}^{1} h(\tau) \Lambda^{\prime}\left(\frac{a_{1} a_{2}}{t b+(1-\tau) a_{1}}\right) \frac{a_{1} a_{2}\left(a_{1}-a_{2}\right)}{\left(t b+(1-\tau) a_{1}\right)^{2}} \mathrm{~d} \tau \\
& =\Lambda(x)-\frac{a_{1} a_{2}}{\left(a_{2}-a_{1}\right)} \int_{a_{1}}^{a_{2}} \frac{\Lambda(s)}{s^{2}} \mathrm{~d} s .
\end{aligned}
$$


where

$$
h(\tau)=\left\{\begin{array}{rr}
\tau, & \tau \in\left[0, \frac{a_{1}\left(a_{2}-x\right)}{x\left(a_{2}-a_{1}\right)}\right], \\
\tau-1, & \tau \in\left(\frac{a_{1}\left(a_{2}-x\right)}{x\left(a_{2}-a_{1}\right)}, 1\right] .
\end{array}\right.
$$

Before proceeding to our next result, let us recall the integral form of the hypergeometric function:

$$
{ }_{2} F_{1}(x, y ; c ; z)=\frac{1}{\mathrm{~B}(y, c-y)} \int_{0}^{1} \tau^{y-1}(1-\tau)^{c-y-1}(1-z t)^{-x} \mathrm{~d} \tau,
$$

for $|z|\langle 1, c\rangle y\rangle 0$.

We now derive our first refinement of Ostrowski type inequality using the class of harmonically convex functions.

Theorem 1. Let the expression $T(\Lambda, \varrho, \zeta, x)$ be as defined in (1) of Lemma 1. Suppose $\Lambda: I \rightarrow \mathbb{R}$ is a differentiable mapping on $I^{\circ}$, where $a_{1}, a_{2} \in I$ with $a_{1}<a_{2}$. If $\left|\Lambda^{\prime}\right|$ is harmonically convex, then, for $\varrho \in[0,1], x \in\left[a_{1}, a_{2}\right]$ and $\varsigma \in[0,1] \backslash\left\{\frac{1}{2}\right\}$, the following inequality holds true:

$$
\begin{aligned}
|T(\Lambda, \varrho, \varsigma, x)| \leq & a_{1} a_{2}\left(a_{2}-a_{1}\right)(1-2 \varsigma)\left\{\left(\left|\Lambda^{\prime}\left(\frac{a_{1} a_{2}}{A_{a_{2}}}\right)\right| B_{1}+\left|\Lambda^{\prime}\left(\frac{a_{1} a_{2}}{A_{a_{1}}}\right)\right| B_{2}\right)\right. \\
& \left.+\left(\left|\Lambda^{\prime}\left(\frac{a_{1} a_{2}}{A_{a_{2}}}\right)\right| B_{3}+\left|\Lambda^{\prime}\left(\frac{a_{1} a_{2}}{A_{a_{1}}}\right)\right| B_{4}\right)\right\},
\end{aligned}
$$

where

$$
\begin{aligned}
B_{1}= & \int_{0}^{\substack{a_{1}\left(a_{2}-x\right) \\
x\left(a_{2}-a_{1}\right)}}\left|\tau-\varrho \frac{a_{1}\left(a_{2}-x\right)}{x\left(a_{2}-a_{1}\right)}\right| \frac{\tau}{A_{\tau}^{2}} \mathrm{~d} \tau \\
= & \left(\varsigma a_{1}+(1-\varsigma) a_{2}\right)^{-2}\left(\frac{a_{1}\left(a_{2}-x\right)}{x\left(a_{2}-a_{1}\right)}\right)^{3}\left\{\frac{\varrho^{3}}{3}{ }_{2} F_{1}\left(2,2,4,\left(\frac{a_{1} \varrho\left(a_{2}-x\right)(1-2 \zeta)}{x\left(u a_{1}+(1-\varsigma) a_{2}\right)}\right)\right)\right. \\
& \left.+\frac{1}{3}{ }_{2} F_{1}\left(2,3,4,\left(\frac{a_{1}\left(a_{2}-x\right)(1-2 \varsigma)}{x\left(u a_{1}+(1-\varsigma) a_{2}\right)}\right)\right)-\frac{\varrho}{2}{ }_{2} F_{1}\left(2,2,3,\left(\frac{a_{1}\left(a_{2}-x\right)(1-2 \varsigma)}{x\left(u a_{1}+(1-\varsigma) a_{2}\right)}\right)\right)\right\}, \\
B_{2}= & \int_{0}^{\frac{a_{1}\left(a_{2}-x\right)}{x\left(a_{2}-a_{1}\right)}}\left|\tau-\varrho \frac{a_{1}\left(a_{2}-x\right)}{x\left(a_{2}-a_{1}\right)}\right| \frac{1-\tau}{A_{\tau}^{2}} \mathrm{~d} \tau \\
= & \left(\varsigma a_{1}+(1-\varsigma) a_{2}\right)^{-2}\left(\frac{a_{1}\left(a_{2}-x\right)}{x\left(a_{2}-a_{1}\right)}\right)^{2}\left\{\varrho^{2}{ }_{2} F_{1}\left(2,1,3,\left(\frac{a_{1} \varrho\left(a_{2}-x\right)(1-2 \varsigma)}{x\left(u a_{1}+(1-\varsigma) a_{2}\right)}\right)\right)\right. \\
& -\frac{\varrho a_{1}\left(a_{2}-x\right)}{6 x\left(a_{2}-a_{1}\right)}{ }_{2} F_{1}\left(2,2,4,\left(\frac{\varrho a_{1}\left(a_{2}-x\right)(1-2 \varsigma)}{x\left(u a_{1}+(1-\varsigma) a_{2}\right)}\right)\right) \\
& +\frac{1}{2}\left(1+\frac{\rho a_{1}\left(a_{2}-x\right)}{x\left(a_{2}-a_{1}\right)}\right){ }_{2} F_{1}\left(2,2,3,\left(\frac{a_{1}\left(a_{2}-x\right)(1-2 \varsigma)}{x\left(u a_{1}+(1-\varsigma) a_{2}\right)}\right)\right) \\
& \left.-\frac{\varrho a_{1}\left(a_{2}-x\right)}{3 x\left(a_{2}-a_{1}\right)}{ }_{2} F_{1}\left(2,3,4,\left(\frac{a_{1}\left(a_{2}-x\right)(1-2 \varsigma)}{x\left(u a_{1}+(1-\varsigma) a_{2}\right)}\right)\right)-\varrho{ }_{2} F_{1}\left(2,1,2,\left(\frac{a_{1}\left(a_{2}-x\right)(1-2 \varsigma)}{x\left(u a_{1}+(1-\varsigma) a_{2}\right)}\right)\right)\right\},
\end{aligned}
$$




$$
\begin{aligned}
B_{3}= & \int_{\frac{a_{1}\left(a_{2}-x\right)}{x\left(a_{2}-a_{1}\right)}}^{1}\left|\tau-1+\varrho \frac{a_{2}\left(x-a_{1}\right)}{x\left(a_{2}-a_{1}\right)}\right| \frac{\tau}{A_{\tau}^{2}} \mathrm{~d} \tau \\
= & \left(\varsigma a_{2}+(1-\varsigma) a_{1}\right)^{-2}\left(\frac{a_{2}\left(x-a_{1}\right)}{x\left(a_{2}-a_{1}\right)}\right)^{2}\left\{\varrho^{2}{ }_{2} F_{1}\left(2,1,3,\left(\frac{a_{2} \varrho\left(x-a_{1}\right)(2 \varsigma-1)}{x\left(u b+(1-\varsigma) a_{1}\right)}\right)\right)\right. \\
& -\frac{\varrho a_{2}\left(x-a_{1}\right)}{6 x\left(a_{2}-a_{1}\right)}{ }_{2} F_{1}\left(2,2,4,\left(\frac{\varrho a_{2}\left(a_{1}-x\right)(1-2 \varsigma)}{x\left(u b+(1-\varsigma) a_{1}\right)}\right)\right) \\
& +\frac{1}{2}\left(1+\frac{\varrho a_{2}\left(a_{1}-x\right)}{x\left(a_{2}-a_{1}\right)}\right){ }_{2} F_{1}\left(2,2,3,\left(\frac{a_{2}\left(a_{1}-x\right)(2 \varsigma-1)}{x\left(u b+(1-\varsigma) a_{1}\right)}\right)\right) \\
& \left.-\frac{\varrho a_{2}\left(x-a_{1}\right)}{3 x\left(a_{2}-a_{1}\right)}{ }_{2} F_{1}\left(2,3,4,\left(\frac{a_{2}\left(x-a_{1}\right)(1-2 \varsigma)}{x\left(u b+(1-\varsigma) a_{1}\right)}\right)\right)-\varrho_{2} F_{1}\left(2,1,2,\left(\frac{a_{2}\left(x-a_{1}\right)(2 \varsigma-1)}{x\left(u b+(1-\varsigma) a_{1}\right)}\right)\right)\right\}, \\
B_{4}= & \int^{\frac{a_{1}\left(a_{2}-x\right)}{x\left(a_{2}-a_{1}\right)}}\left|\tau-1+\varrho \frac{a_{2}\left(x-a_{1}\right)}{x\left(a_{2}-a_{1}\right)}\right| \frac{1-\tau}{A_{\tau}^{2}} \mathrm{~d} \tau \\
= & \left(\varsigma a_{2}+(1-\varsigma) a_{1}\right)^{-2}\left(\frac{a_{2}\left(x-a_{1}\right)}{x\left(a_{2}-a_{1}\right)}\right)^{3}\left\{\frac{\varrho^{3}}{3}{ }_{2} F_{1}\left(2,2,4,\left(\frac{a_{2} \varrho\left(x-a_{1}\right)(2 \varsigma-1)}{x\left(u b+(1-\varsigma) a_{1}\right)}\right)\right)\right. \\
& \left.+\frac{1}{3}{ }_{2} F_{1}\left(2,3,4,\left(\frac{a_{2}\left(a_{1}-x\right)(2 \varsigma-1)}{x\left(u b+(1-\varsigma) a_{1}\right)}\right)\right)-\frac{\varrho}{2}{ }_{2} F_{1}\left(2,2,3,\left(\frac{a_{2}\left(x-a_{1}\right)(2 \varsigma-1)}{x\left(u b+(1-\varsigma) a_{1}\right)}\right)\right)\right\} .
\end{aligned}
$$

Proof. For the sake of brevity, we write $A_{a_{1}}=\varsigma a_{1}+(1-\varsigma) a_{2}$ and $A_{a_{2}}=\varsigma a_{2}+(1-\varsigma) a_{1}$. Using Lemma 1, we have

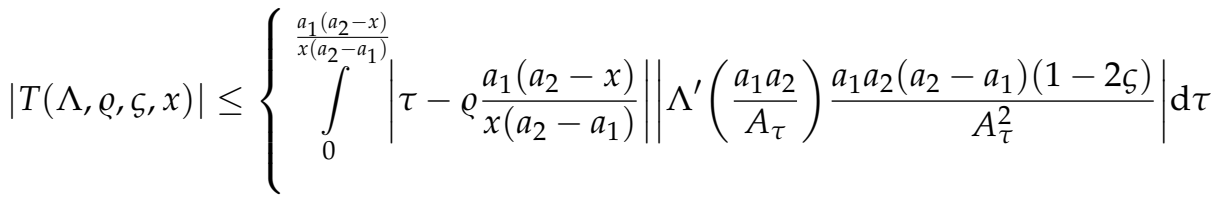

$$
\begin{aligned}
& \left.+\int_{\frac{a_{1}\left(a_{2}-x\right)}{x\left(a_{2}-a_{1}\right)}}^{1}\left|\tau-1+\varrho \frac{a_{2}\left(x-a_{1}\right)}{x\left(a_{2}-a_{1}\right)}\right|\left|\Lambda^{\prime}\left(\frac{a_{1} a_{2}}{A_{\tau}}\right) \frac{a_{1} a_{2}\left(a_{2}-a_{1}\right)(1-2 \varsigma)}{A_{\tau}^{2}}\right| \mathrm{d} \tau\right\} \\
& \leq a_{1} a_{2}\left(a_{2}-a_{1}\right)(1-2 \varsigma)\left\{\int_{0}^{\frac{a_{1}\left(a_{2}-x\right)}{x\left(a_{2}-a_{1}\right)}}\left|\tau-\varrho \frac{a_{1}\left(a_{2}-x\right)}{x\left(a_{2}-a_{1}\right)}\right|\left|\Lambda^{\prime}\left(\frac{a_{1} a_{2}}{A_{\tau}}\right) \frac{1}{A_{\tau}^{2}}\right| \mathrm{d} \tau\right. \\
& \left.+\int_{\frac{a_{1}\left(a_{2}-x\right)}{x\left(a_{2}-a_{1}\right)}}^{1}\left|\tau-1+\varrho \frac{a_{2}\left(x-a_{1}\right)}{x\left(a_{2}-a_{1}\right)}\right|\left|\Lambda^{\prime}\left(\frac{a_{1} a_{2}}{A_{\tau}}\right) \frac{1}{A_{\tau}^{2}}\right| \mathrm{d} \tau\right\} \\
& =a_{1} a_{2}\left(a_{2}-a_{1}\right)(1-2 \varsigma) K_{1} \text {. }
\end{aligned}
$$


Using the harmonic convexity of $\left|\Lambda^{\prime}\right|$, we get

$$
\begin{aligned}
K_{1} \leq & \left\{\left|\Lambda^{\prime}\left(\frac{a_{1} a_{2}}{A_{a_{2}}}\right)\right| \int_{0}^{\frac{a_{1}\left(a_{2}-x\right)}{x\left(a_{2}-a_{1}\right)}}\left|\tau-\varrho \frac{a_{1}\left(a_{2}-x\right)}{x\left(a_{2}-a_{1}\right)}\right| \frac{\tau}{A_{\tau}} \mathrm{d} \tau\right. \\
& \left.+\left|\Lambda^{\prime}\left(\frac{a_{1} a_{2}}{A_{a_{1}}}\right)\right| \int_{0}^{\frac{a_{1}\left(a_{2}-x\right)}{x\left(a_{2}-a_{1}\right)}}\left|\tau-\varrho \frac{a_{1}\left(a_{2}-x\right)}{x\left(a_{2}-a_{1}\right)}\right| \frac{(1-\tau)}{A_{\tau}} \mathrm{d} \tau\right) \\
& +|| \Lambda^{\prime}\left(\frac{a_{1} a_{2}}{A_{a_{2}}}\right)\left|\int_{\frac{a_{1}\left(a_{2}-x\right)}{x\left(a_{2}-a_{1}\right)}}^{1}\right| \tau-1+\varrho \frac{a_{2}\left(x-a_{1}\right)}{x\left(a_{2}-a_{1}\right)} \mid \frac{\tau}{A_{\tau}} \mathrm{d} \tau \\
& \left.\left.+\left|\Lambda^{\prime}\left(\frac{a_{1} a_{2}}{A_{a_{1}}}\right)\right| \int_{\frac{a_{1}\left(a_{2}-x\right)}{x\left(a_{2}-a_{1}\right)}}^{1}\left|\tau-1+\varrho \frac{a_{2}\left(x-a_{1}\right)}{x\left(a_{2}-a_{1}\right)}\right| \frac{(1-\tau)}{A_{\tau}} \mathrm{d} \tau\right)\right\} \\
= & \left\{\left(\left|\Lambda^{\prime}\left(\frac{a_{1} a_{2}}{A_{a_{2}}}\right)\right| B_{1}+\left|\Lambda^{\prime}\left(\frac{a_{1} a_{2}}{A_{a_{1}}}\right)\right| B_{2}\right)+\left(\left|\Lambda^{\prime}\left(\frac{a_{1} a_{2}}{A_{a_{2}}}\right)\right| B_{3}+\left|\Lambda^{\prime}\left(\frac{a_{1} a_{2}}{A_{a_{1}}}\right)\right| B_{4}\right)\right\} .
\end{aligned}
$$

This completes the proof.

We now derive a second refinement of Ostrowski type inequalities involving harmonically convex functions. We derive this result using the power mean inequality.

Theorem 2. Let $\Lambda: I \rightarrow \mathbb{R}$ be a differentiable mapping on $I^{\circ}$, where $a_{1}, a_{2} \in I$ with $a_{1}<a_{2}$. If, for $q \geq 1,\left|\Lambda^{\prime}\right|^{q}$ is harmonically convex, for $\varrho \in[0,1], x \in\left[a_{1}, a_{2}\right]$ and $\varsigma \in[0,1] \backslash\left\{\frac{1}{2}\right\}$, the following inequality holds true:

$$
\begin{aligned}
|T(\Lambda, \varrho, \varsigma, x)| \leq & a_{1} a_{2}\left(a_{2}-a_{1}\right)(1-2 \varsigma)\left(\left(\phi_{1}\right)^{1-\frac{1}{q}}\left(\left|\Lambda^{\prime}\left(\frac{a_{1} a_{2}}{A_{a_{2}}}\right)\right|^{q} B_{1}^{*}+\left|\Lambda^{\prime}\left(\frac{a_{1} a_{2}}{A_{a_{1}}}\right)\right|^{q} B_{2}^{*}\right)^{\frac{1}{q}}\right. \\
& \left.+\left(\phi_{2}\right)^{1-\frac{1}{q}}\left(\left|\Lambda^{\prime}\left(\frac{a_{1} a_{2}}{A_{a_{2}}}\right)\right|^{q} B_{3}^{*}+\left|\Lambda^{\prime}\left(\frac{a_{1} a_{2}}{A_{a_{1}}}\right)\right|^{q} B_{4}^{*}\right)^{\frac{1}{q}}\right)
\end{aligned}
$$

where

$$
\begin{aligned}
& \phi_{1}=\int_{0}^{\frac{a_{1}\left(a_{2}-x\right)}{x\left(a_{2}-a_{1}\right)}}\left|\tau-\varrho \frac{a_{1}\left(a_{2}-x\right)}{x\left(a_{2}-a_{1}\right)}\right| \mathrm{d} \tau=\left(\frac{\left(2 \varrho^{2}-2 \varrho+1\right)}{2}\right)\left(\frac{a_{1}\left(a_{2}-x\right)}{x\left(a_{2}-a_{1}\right)}\right)^{2},
\end{aligned}
$$

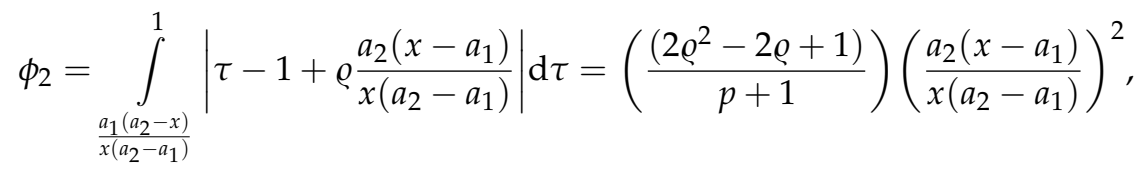


Symmetry 2021, 13, 201

8 of 23

$$
\begin{aligned}
& B_{1}^{*}=\int_{0}^{\frac{a_{1}\left(a_{2}-x\right)}{x\left(a_{2}-a_{1}\right)}}\left|\tau-\varrho \frac{a_{1}\left(a_{2}-x\right)}{x\left(a_{2}-a_{1}\right)}\right| \frac{\tau}{\left(A_{\tau}\right)^{2 q}} \mathrm{~d} \tau \\
& =\left(\varsigma a_{1}+(1-\varsigma) a_{2}\right)^{-2}\left(\frac{a_{1}\left(a_{2}-x\right)}{x\left(a_{2}-a_{1}\right)}\right)^{3}\left\{\frac{\varrho^{3}}{3}{ }_{2} F_{1}\left(2 q, 2,4,\left(\frac{a_{1} \varrho\left(a_{2}-x\right)(1-2 \zeta)}{x\left(u a_{1}+(1-\varsigma) a_{2}\right)}\right)\right)\right. \\
& \left.+\frac{1}{3}{ }_{2} F_{1}\left(2 q, 3,4,\left(\frac{a_{1}\left(a_{2}-x\right)(1-2 \varsigma)}{x\left(u a_{1}+(1-\varsigma) a_{2}\right)}\right)\right)-\frac{\varrho}{2}{ }_{2} F_{1}\left(2 q, 2,3,\left(\frac{a_{1}\left(a_{2}-x\right)(1-2 \varsigma)}{x\left(u a_{1}+(1-\varsigma) a_{2}\right)}\right)\right)\right\}, \\
& B_{2}^{*}=\int_{0}^{\frac{a_{1}\left(a_{2}-x\right)}{x\left(a_{2}-a_{1}\right)}}\left|\tau-\varrho \frac{a_{1}\left(a_{2}-x\right)}{x\left(a_{2}-a_{1}\right)}\right| \frac{1-\tau}{\left(A_{\tau}\right)^{2 q}} \mathrm{~d} \tau \\
& =\left(\varsigma a_{1}+(1-\varsigma) a_{2}\right)^{-2}\left(\frac{a_{1}\left(a_{2}-x\right)}{x\left(a_{2}-a_{1}\right)}\right)^{2}\left\{\varrho^{2}{ }_{2} F_{1}\left(2 q, 1,3,\left(\frac{a_{1} \varrho\left(a_{2}-x\right)(1-2 \varsigma)}{x\left(u a_{1}+(1-\varsigma) a_{2}\right)}\right)\right)\right. \\
& -\frac{\rho a_{1}\left(a_{2}-x\right)}{6 x\left(a_{2}-a_{1}\right)}{ }_{2} F_{1}\left(2 q, 2,4,\left(\frac{\rho a_{1}\left(a_{2}-x\right)(1-2 \varsigma)}{x\left(u a_{1}+(1-\varsigma) a_{2}\right)}\right)\right) \\
& +\frac{1}{2}\left(1+\frac{\varrho a_{1}\left(a_{2}-x\right)}{x\left(a_{2}-a_{1}\right)}\right){ }_{2} F_{1}\left(2 q, 2,3,\left(\frac{a_{1}\left(a_{2}-x\right)(1-2 \varsigma)}{x\left(u a_{1}+(1-\varsigma) a_{2}\right)}\right)\right) \\
& \left.-\frac{\varrho a_{1}\left(a_{2}-x\right)}{3 x\left(a_{2}-a_{1}\right)}{ }_{2} F_{1}\left(2 q, 3,4,\left(\frac{a_{1}\left(a_{2}-x\right)(1-2 \zeta)}{x\left(u a_{1}+(1-\varsigma) a_{2}\right)}\right)\right)-\varrho_{2} F_{1}\left(2 q, 1,2,\left(\frac{a_{1}\left(a_{2}-x\right)(1-2 \varsigma)}{x\left(u a_{1}+(1-\varsigma) a_{2}\right)}\right)\right)\right\},
\end{aligned}
$$

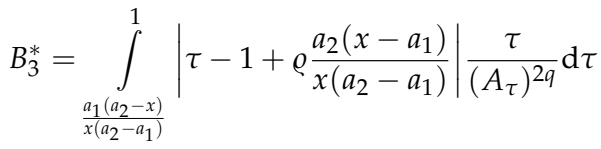

$$
\begin{aligned}
& =\left(\varsigma a_{2}+(1-\varsigma) a_{1}\right)^{-2}\left(\frac{a_{2}\left(x-a_{1}\right)}{x\left(a_{2}-a_{1}\right)}\right)^{2}\left\{\varrho^{2}{ }_{2} F_{1}\left(2 q, 1,3,\left(\frac{a_{2} \varrho\left(x-a_{1}\right)(2 \varsigma-1)}{x\left(u b+(1-\varsigma) a_{1}\right)}\right)\right)\right. \\
& -\frac{\varrho a_{2}\left(x-a_{1}\right)}{6 x\left(a_{2}-a_{1}\right)}{ }_{2} F_{1}\left(2 q, 2,4,\left(\frac{\varrho a_{2}\left(a_{1}-x\right)(1-2 \varsigma)}{x\left(u b+(1-\varsigma) a_{1}\right)}\right)\right) \\
& +\frac{1}{2}\left(1+\frac{\varrho a_{2}\left(a_{1}-x\right)}{x\left(a_{2}-a_{1}\right)}\right){ }_{2} F_{1}\left(2 q, 2,3,\left(\frac{a_{2}\left(a_{1}-x\right)(2 \varsigma-1)}{x\left(u b+(1-\varsigma) a_{1}\right)}\right)\right) \\
& \left.-\frac{\rho a_{2}\left(x-a_{1}\right)}{3 x\left(a_{2}-a_{1}\right)}{ }_{2} F_{1}\left(2 q, 3,4,\left(\frac{a_{2}\left(x-a_{1}\right)(1-2 \varsigma)}{x\left(u b+(1-\varsigma) a_{1}\right)}\right)\right)-\varrho_{2} F_{1}\left(2 q, 1,2,\left(\frac{a_{2}\left(x-a_{1}\right)(2 \varsigma-1)}{x\left(u b+(1-\varsigma) a_{1}\right)}\right)\right)\right\}, \\
& B_{4}^{*}=\int_{\frac{a_{1}\left(a_{2}-x\right)}{x\left(a_{2}-a_{1}\right)}}^{1}\left|\tau-1+\varrho \frac{a_{2}\left(x-a_{1}\right)}{x\left(a_{2}-a_{1}\right)}\right| \frac{1-\tau}{\left(A_{\tau}\right)^{2 q}} \mathrm{~d} \tau \\
& =\left(\varsigma a_{2}+(1-\varsigma) a_{1}\right)^{-2}\left(\frac{a_{2}\left(x-a_{1}\right)}{x\left(a_{2}-a_{1}\right)}\right)^{3}\left\{\frac{\varrho^{3}}{3}{ }_{2} F_{1}\left(2 q, 2,4,\left(\frac{a_{2} \varrho\left(x-a_{1}\right)(2 \varsigma-1)}{x\left(u b+(1-\varsigma) a_{1}\right)}\right)\right)\right. \\
& \left.+\frac{1}{3}{ }_{2} F_{1}\left(2 q, 3,4,\left(\frac{a_{2}\left(a_{1}-x\right)(2 \varsigma-1)}{x\left(u b+(1-\varsigma) a_{1}\right)}\right)\right)-\frac{\varrho}{2}{ }_{2} F_{1}\left(2 q, 2,3,\left(\frac{a_{2}\left(x-a_{1}\right)(2 \varsigma-1)}{x\left(u b+(1-\varsigma) a_{1}\right)}\right)\right)\right\} \text {. }
\end{aligned}
$$


Proof. For the sake of brevity, we write $A_{a_{1}}=\varsigma a_{1}+(1-\varsigma) a_{2}$ and $A_{a_{2}}=\varsigma a_{2}+(1-\varsigma) a_{1}$. We suppose that $q>1$. Taking modulus in Lemma 1 and using the power mean inequality, we have

$$
\begin{aligned}
& |T(\Lambda, \varrho, \zeta, x)| \leq\left\{\int_{0}^{\frac{a_{1}\left(a_{2}-x\right)}{x\left(a_{2}-a_{1}\right)}}\left|\tau-\varrho \frac{a_{1}\left(a_{2}-x\right)}{x\left(a_{2}-a_{1}\right)}\right|\left|\Lambda^{\prime}\left(\frac{a_{1} a_{2}}{A_{\tau}}\right) \frac{a_{1} a_{2}\left(a_{2}-a_{1}\right)(1-2 \varsigma)}{A_{\tau}^{2}}\right| \mathrm{d} \tau\right. \\
& \left.+\int_{\frac{a_{1}\left(a_{2}-x\right)}{x\left(a_{2}-a_{1}\right)}}^{1}\left|\tau-1+\varrho \frac{a_{2}\left(x-a_{1}\right)}{x\left(a_{2}-a_{1}\right)}\right|\left|\Lambda^{\prime}\left(\frac{a_{1} a_{2}}{A_{\tau}}\right) \frac{a_{1} a_{2}\left(a_{2}-a_{1}\right)(1-2 \zeta)}{A_{\tau}^{2}}\right| \mathrm{d} \tau\right\} \\
& \leq a_{1} a_{2}\left(a_{2}-a_{1}\right)(1-2 \varsigma)\left\{\left(\int_{0}^{\frac{a_{1}\left(a_{2}-x\right)}{x\left(a_{2}-a_{1}\right)}}\left|\tau-\varrho \frac{a_{1}\left(a_{2}-x\right)}{x\left(a_{2}-a_{1}\right)}\right| \mathrm{d} \tau\right)^{1-\frac{1}{q}}\right. \\
& \times\left(\int_{0}^{\frac{a_{1}\left(a_{2}-x\right)}{x\left(a_{2}-a_{1}\right)}}\left|\tau-\varrho \frac{a_{1}\left(a_{2}-x\right)}{x\left(a_{2}-a_{1}\right)}\right|\left|\Lambda^{\prime}\left(\frac{a_{1} a_{2}}{A_{\tau}}\right) \frac{1}{A_{\tau}^{2}}\right|^{q} \mathrm{~d} \tau\right)^{\frac{1}{q}} \\
& +\left(\int_{\frac{a_{1}\left(a_{2}-x\right)}{x\left(a_{2}-a_{1}\right)}}^{1}\left|\tau-1+\varrho \frac{a_{2}\left(x-a_{1}\right)}{x\left(a_{2}-a_{1}\right)}\right| \mathrm{d} \tau\right)^{1-\frac{1}{q}} \\
& \left.\times\left(\int_{\frac{a_{1}\left(a_{2}-x\right)}{x\left(a_{2}-a_{1}\right)}}^{1}\left|\tau-1+\varrho \frac{a_{2}\left(x-a_{1}\right)}{x\left(a_{2}-a_{1}\right)}\right|\left|\Lambda^{\prime}\left(\frac{a_{1} a_{2}}{A_{\tau}}\right) \frac{1}{A_{\tau}^{2}}\right|^{q} \mathrm{~d} \tau\right)^{\frac{1}{q}}\right\} \\
& =a_{1} a_{2}\left(a_{2}-a_{1}\right)(1-2 \varsigma) K_{2} \text {. }
\end{aligned}
$$


Using the harmonic convexity of $\left|\Lambda^{\prime}\right|^{q}$, we get

$$
\begin{aligned}
& K_{2} \leq\left\{\left(\int_{0}^{\frac{a_{1}\left(a_{2}-x\right)}{x\left(a_{2}-a_{1}\right)}}\left|\tau-\varrho \frac{a_{1}\left(a_{2}-x\right)}{x\left(a_{2}-a_{1}\right)}\right| \mathrm{d} \tau\right)^{1-\frac{1}{q}}\right. \\
& \times\left(\left|\Lambda^{\prime}\left(\frac{a_{1} a_{2}}{A_{a_{2}}}\right)\right|^{q} \int_{0}^{\frac{a_{1}\left(a_{2}-x\right)}{x\left(a_{2}-a_{1}\right)}}\left|\tau-\varrho \frac{a_{1}\left(a_{2}-x\right)}{x\left(a_{2}-a_{1}\right)}\right| \frac{\tau}{\left(A_{\tau}\right)^{2 q}} \mathrm{~d} \tau\right. \\
& \left.+\left|\Lambda^{\prime}\left(\frac{a_{1} a_{2}}{A_{a_{1}}}\right)\right|^{q} \int_{0}^{\frac{a_{1}\left(a_{2}-x\right)}{x\left(a_{2}-a_{1}\right)}}\left|\tau-\varrho \frac{a_{1}\left(a_{2}-x\right)}{x\left(a_{2}-a_{1}\right)}\right| \frac{(1-\tau)}{\left(A_{\tau}\right)^{2 q}} \mathrm{~d} \tau\right)^{\frac{1}{q}} \\
& +\left(\int_{\frac{a_{1}\left(a_{2}-x\right)}{x\left(a_{2}-a_{1}\right)}}^{1}\left|\tau-1+\varrho \frac{a_{2}\left(x-a_{1}\right)}{x\left(a_{2}-a_{1}\right)}\right| \mathrm{d} \tau\right)^{1-\frac{1}{q}} \\
& \times\left(\left|\Lambda^{\prime}\left(\frac{a_{1} a_{2}}{A_{a_{2}}}\right)\right|^{q} \int_{\frac{a_{1}\left(a_{2}-x\right)}{x\left(a_{2}-a_{1}\right)}}^{1}\left|\tau-1+\varrho \frac{a_{2}\left(x-a_{1}\right)}{x\left(a_{2}-a_{1}\right)}\right| \frac{\tau}{\left(A_{\tau}\right)^{2 q}} \mathrm{~d} \tau\right. \\
& \left.\left.+\left|\Lambda^{\prime}\left(\frac{a_{1} a_{2}}{A_{a_{1}}}\right)\right|^{q} \int_{\frac{a_{1}\left(a_{2}-x\right)}{x\left(a_{2}-a_{1}\right)}}^{1}\left|\tau-1+\varrho \frac{a_{2}\left(x-a_{1}\right)}{x\left(a_{2}-a_{1}\right)}\right| \frac{(1-\tau)}{\left(A_{\tau}\right)^{2 q}} \mathrm{~d} \tau\right)^{\frac{1}{q}}\right\} \\
& =\left(\left(\phi_{1}\right)^{1-\frac{1}{q}}\left(\left|\Lambda^{\prime}\left(\frac{a_{1} a_{2}}{A_{a_{2}}}\right)\right|^{q} B_{1}^{*}+\left|\Lambda^{\prime}\left(\frac{a_{1} a_{2}}{A_{a_{1}}}\right)\right|^{q} B_{2}^{*}\right)^{\frac{1}{q}}\right. \\
& \left.+\left(\phi_{2}\right)^{1-\frac{1}{q}}\left(\left|\Lambda^{\prime}\left(\frac{a_{1} a_{2}}{A_{a_{2}}}\right)\right|^{q} B_{3}^{*}+\left|\Lambda^{\prime}\left(\frac{a_{1} a_{2}}{A_{a_{1}}}\right)\right|^{q} B_{4}^{*}\right)^{\frac{1}{q}}\right) .
\end{aligned}
$$

This completes the proof.

Remark 1. If $q=1$, then Theorem 2 reduces to Theorem 1.

Corollary 1. Under the assumptions of Theorem 2, if we choose $\varsigma=0$ in (4), then we have

$$
\begin{aligned}
|T(\Lambda, \varrho, 0, x)| \leq & a_{1} a_{2}\left(a_{2}-a_{1}\right)\left(\left(\phi_{1}\right)^{1-\frac{1}{q}}\left(\left|\Lambda^{\prime}\left(\frac{a_{1} a_{2}}{A_{a_{2}}}\right)\right|^{q} B_{1}^{* *}+\left|\Lambda^{\prime}\left(\frac{a_{1} a_{2}}{A_{a_{1}}}\right)\right|^{q} B_{2}^{* *}\right)^{\frac{1}{q}}\right. \\
& \left.+\left(\phi_{2}\right)^{1-\frac{1}{q}}\left(\left|\Lambda^{\prime}\left(\frac{a_{1} a_{2}}{A_{a_{2}}}\right)\right|^{q} B_{3}^{* *}+\left|\Lambda^{\prime}\left(\frac{a_{1} a_{2}}{A_{a_{1}}}\right)\right|^{q} B_{4}^{* *}\right)^{\frac{1}{q}}\right)
\end{aligned}
$$


where $\phi_{1}, \phi_{2}$ are given in Theorem 2 and

$$
\begin{aligned}
& B_{1}^{* *}=\int_{0}^{\frac{a_{1}\left(a_{2}-x\right)}{x\left(a_{2}-a_{1}\right)}}\left|\tau-\varrho \frac{a_{1}\left(a_{2}-x\right)}{x\left(a_{2}-a_{1}\right)}\right| \frac{\tau}{\left(\tau a_{1}+(1-\tau) a_{2}\right)^{2 q}} \mathrm{~d} \tau \\
& =a_{2}{ }^{-2}\left(\frac{a_{1}\left(a_{2}-x\right)}{x\left(a_{2}-a_{1}\right)}\right)^{3}\left\{\frac{\varrho^{3}}{3}{ }_{2} F_{1}\left(2 q, 2,4,\left(\frac{a_{1} \varrho\left(a_{2}-x\right)}{x b}\right)\right)\right. \\
& \left.+\frac{1}{3}{ }_{2} F_{1}\left(2 q, 3,4,\left(\frac{a_{1}\left(a_{2}-x\right)}{x b}\right)\right)-\frac{\varrho}{2}{ }_{2} F_{1}\left(2 q, 2,3,\left(\frac{a_{1}\left(a_{2}-x\right)}{x b}\right)\right)\right\}, \\
& B_{2}^{* *}=\int_{0}^{\frac{a_{1}\left(a_{2}-x\right)}{x\left(a_{2}-a_{1}\right)}}\left|\tau-\varrho \frac{a_{1}\left(a_{2}-x\right)}{x\left(a_{2}-a_{1}\right)}\right| \frac{1-\tau}{\left(\tau a_{1}+(1-\tau) a_{2}\right)^{2 q}} \mathrm{~d} \tau \\
& =a_{2}{ }^{-2}\left(\frac{a_{1}\left(a_{2}-x\right)}{x\left(a_{2}-a_{1}\right)}\right)^{2}\left\{\varrho^{2}{ }_{2} F_{1}\left(2 q, 1,3,\left(\frac{a_{1} \varrho\left(a_{2}-x\right)}{x b}\right)\right)\right. \\
& -\frac{\varrho a_{1}\left(a_{2}-x\right)}{6 x\left(a_{2}-a_{1}\right)}{ }_{2} F_{1}\left(2 q, 2,4,\left(\frac{\varrho a_{1}\left(a_{2}-x\right)}{x a}\right)\right) \\
& +\frac{1}{2}\left(1+\frac{\varrho a_{1}\left(a_{2}-x\right)}{x\left(a_{2}-a_{1}\right)}\right){ }_{2} F_{1}\left(2 q, 2,3,\left(\frac{a_{1}\left(a_{2}-x\right)}{x b}\right)\right) \\
& \left.-\frac{\varrho a_{1}\left(a_{2}-x\right)}{3 x\left(a_{2}-a_{1}\right)}{ }_{2} F_{1}\left(2 q, 3,4,\left(\frac{a_{1}\left(a_{2}-x\right)}{x b}\right)\right)-\varrho_{2} F_{1}\left(2 q, 1,2,\left(\frac{a_{1}\left(a_{2}-x\right)}{x b}\right)\right)\right\}, \\
& B_{3}^{* *}=\int_{\frac{a_{1}\left(a_{2}-x\right)}{x\left(a_{2}-a_{1}\right)}}^{1}\left|\tau-1+\varrho \frac{a_{2}\left(x-a_{1}\right)}{x\left(a_{2}-a_{1}\right)}\right| \frac{\tau}{\left(\tau a_{1}+(1-\tau) a_{2}\right)^{2 q}} \mathrm{~d} \tau \\
& =a_{1}{ }^{-2}\left(\frac{a_{2}\left(x-a_{1}\right)}{x\left(a_{2}-a_{1}\right)}\right)^{2}\left\{\varrho^{2}{ }_{2} F_{1}\left(2 q, 1,3,\left(\frac{a_{2} \varrho\left(a_{1}-x\right)}{x a}\right)\right)\right. \\
& -\frac{\rho a_{2}\left(x-a_{1}\right)}{6 x\left(a_{2}-a_{1}\right)}{ }_{2} F_{1}\left(2 q, 2,4,\left(\frac{\rho a_{2}\left(a_{1}-x\right)}{x a}\right)\right) \\
& +\frac{1}{2}\left(1+\frac{\rho a_{2}\left(a_{1}-x\right)}{x\left(a_{2}-a_{1}\right)}\right){ }_{2} F_{1}\left(2 q, 2,3,\left(\frac{a_{2}\left(a_{1}-x\right)}{x a}\right)\right) \\
& \left.-\frac{\varrho a_{2}\left(x-a_{1}\right)}{3 x\left(a_{2}-a_{1}\right)}{ }_{2} F_{1}\left(2 q, 3,4,\left(\frac{a_{2}\left(a_{1}-x\right)}{x a}\right)\right)-\varrho_{2} F_{1}\left(2 q, 1,2,\left(\frac{a_{2}\left(a_{1}-x\right)}{x a}\right)\right)\right\} \text {, } \\
& B_{4}^{* *}=\int_{\frac{a_{1}\left(a_{2}-x\right)}{x\left(a_{2}-a_{1}\right)}}^{1}\left|\tau-1+\varrho \frac{a_{2}\left(a_{1}-x\right)}{x\left(a_{2}-a_{1}\right)}\right| \frac{1-\tau}{\left(\tau a_{1}+(1-\tau) a_{2}\right)^{2 q}} \mathrm{~d} \tau \\
& =a_{1}{ }^{-2}\left(\frac{a_{2}\left(x-a_{1}\right)}{x\left(a_{2}-a_{1}\right)}\right)^{3}\left\{\frac{\varrho^{3}}{3}{ }_{2} F_{1}\left(2 q, 2,4,\left(\frac{a_{2} \varrho\left(a_{1}-x\right)}{x a}\right)\right)\right. \\
& \left.+\frac{1}{3}{ }_{2} F_{1}\left(2 q, 3,4,\left(\frac{a_{2}\left(a_{1}-x\right)}{x a}\right)\right)-\frac{\varrho}{2}{ }_{2} F_{1}\left(2 q, 2,3,\left(\frac{a_{2}\left(a_{1}-x\right)}{x a}\right)\right)\right\} \text {. }
\end{aligned}
$$

Corollary 2. Under the assumptions of Theorem 2, if we choose $\varrho=0$ in (4), then

$$
\begin{aligned}
|T(\Lambda, 0, \zeta, x)| \leq & a_{1} a_{2}\left(a_{2}-a_{1}\right)(1-2 \zeta)\left(\left(\phi_{1}^{* *}\right)^{1-\frac{1}{q}}\left(\left|\Lambda^{\prime}\left(\frac{a_{1} a_{2}}{A_{a_{2}}}\right)\right|^{q} B_{1}^{* * *}+\left|\Lambda^{\prime}\left(\frac{a_{1} a_{2}}{A_{a_{1}}}\right)\right|^{q} B_{2}^{* * *}\right)^{\frac{1}{q}}\right. \\
& \left.+\left(\phi_{2}^{* *}\right)^{1-\frac{1}{q}}\left(\left|\Lambda^{\prime}\left(\frac{a_{1} a_{2}}{A_{a_{2}}}\right)\right|^{q} B_{3}^{* * *}+\left|\Lambda^{\prime}\left(\frac{a_{1} a_{2}}{A_{a_{1}}}\right)\right|^{q} B_{4}^{* * *}\right)^{\frac{1}{q}}\right)
\end{aligned}
$$


where

$$
\begin{aligned}
& \phi_{1}^{* *}=\int_{0}^{\frac{a_{1}\left(a_{2}-x\right)}{x\left(a_{2}-a_{1}\right)}}|\tau| \mathrm{d} \tau=\frac{1}{2}\left(\frac{a_{1}\left(a_{2}-x\right)}{x\left(a_{2}-a_{1}\right)}\right)^{2},
\end{aligned}
$$

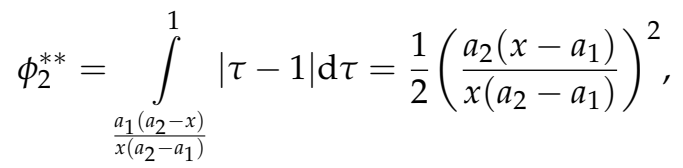

$$
\begin{aligned}
& \frac{a_{1}\left(a_{2}-x\right)}{x\left(a_{2}-a_{1}\right)} \\
& B_{1}^{* * *}=\int_{0}|\tau| \frac{\tau}{\left(A_{\tau}\right)^{2 q}} \mathrm{~d} \tau \\
& =\left(\varsigma a_{1}+(1-\varsigma) a_{2}\right)^{-2}\left(\frac{a_{1}\left(a_{2}-x\right)}{x\left(a_{2}-a_{1}\right)}\right)^{3} \frac{1}{3}{ }_{2} F_{1}\left(2 q, 3,4,\left(\frac{a_{1}\left(a_{2}-x\right)(1-2 \varsigma)}{x\left(u a_{1}+(1-\varsigma) a_{2}\right)}\right)\right), \\
& B_{2}^{* * *}=\int_{0}^{\frac{a_{1}\left(a_{2}-x\right)}{x\left(a_{2}-a_{1}\right)}}|\tau| \frac{1-\tau}{\left(A_{\tau}\right)^{2 q}} \mathrm{~d} \tau \\
& =\left(\varsigma a_{1}+(1-\varsigma) a_{2}\right)^{-2}\left(\frac{a_{1}\left(a_{2}-x\right)}{x\left(a_{2}-a_{1}\right)}\right)^{2} \frac{1}{2}{ }_{2} F_{1}\left(2 q, 2,3,\left(\frac{a_{1}\left(a_{2}-x\right)(1-2 \varsigma)}{x\left(u a_{1}+(1-\varsigma) a_{2}\right)}\right)\right), \\
& B_{3}^{* * *}=\int_{\frac{a_{1}\left(a_{2}-x\right)}{x\left(a_{2}-a_{1}\right)}}^{1}|\tau-1| \frac{\tau}{\left(A_{\tau}\right)^{2 q}} \mathrm{~d} \tau \\
& =\left(\varsigma a_{2}+(1-\varsigma) a_{1}\right)^{-2}\left(\frac{a_{2}\left(x-a_{1}\right)}{x\left(a_{2}-a_{1}\right)}\right)^{2} \frac{1}{2}{ }_{2} F_{1}\left(2 q, 2,3,\left(\frac{a_{2}\left(a_{1}-x\right)(2 \varsigma-1)}{x\left(u b+(1-\varsigma) a_{1}\right)}\right)\right), \\
& B_{4}^{* * *}=\int_{\frac{a_{1}\left(a_{2}-x\right)}{x\left(a_{2}-a_{1}\right)}}^{1}|\tau-1| \frac{1-\tau}{\left(A_{\tau}\right)^{2 q}} \mathrm{~d} \tau \\
& =\left(\varsigma a_{2}+(1-\varsigma) a_{1}\right)^{-2}\left(\frac{a_{2}\left(x-a_{1}\right)}{x\left(a_{2}-a_{1}\right)}\right)^{3} \frac{1}{3}{ }_{2} F_{1}\left(2 q, 3,4,\left(\frac{a_{2}\left(a_{1}-x\right)(2 \varsigma-1)}{x\left(u b+(1-\varsigma) a_{1}\right)}\right)\right) .
\end{aligned}
$$

Corollary 3. Under the assumptions of Theorem 2, if we choose $\varrho=0$ and $x=\frac{2 a_{1} a_{2}}{a_{1}+a_{2}}$ in Corollary 2, then

$$
\begin{aligned}
\left|T\left(\Lambda, 0, \zeta, \frac{2 a_{1} a_{2}}{a_{1}+a_{2}}\right)\right| \leq & a_{1} a_{2}\left(a_{2}-a_{1}\right)(1-2 \varsigma)\left(\left(\phi_{1}^{\diamond}\right)^{1-\frac{1}{q}}\left(\left|\Lambda^{\prime}\left(\frac{a_{1} a_{2}}{A_{a_{2}}}\right)\right|^{q} B_{1}^{\diamond}+\left|\Lambda^{\prime}\left(\frac{a_{1} a_{2}}{A_{a_{1}}}\right)\right|^{q} B_{2}^{\diamond}\right)^{\frac{1}{q}}\right. \\
& \left.+\left(\phi_{2}^{\diamond}\right)^{1-\frac{1}{q}}\left(\left|\Lambda^{\prime}\left(\frac{a_{1} a_{2}}{A_{a_{2}}}\right)\right|^{q} B_{3}^{\diamond}+\left|\Lambda^{\prime}\left(\frac{a_{1} a_{2}}{A_{a_{1}}}\right)\right|^{q} B_{4}^{\diamond}\right)^{\frac{1}{q}}\right)
\end{aligned}
$$

where

$$
\phi_{1}^{\diamond}=\frac{1}{8}, \quad \phi_{2}^{\diamond}=-\frac{1}{8}
$$




$$
\begin{aligned}
& B_{1}^{\diamond}=\left(\varsigma a_{1}+(1-\varsigma) a_{2}\right)^{-2} \frac{1}{24}{ }_{2} F_{1}\left(2 q, 3,4, \frac{\left(a_{2}-a_{1}\right)(1-2 \varsigma)}{2\left(u a_{1}+(1-\varsigma) a_{2}\right)}\right), \\
& B_{2}^{\diamond}=\left(\varsigma a_{1}+(1-\varsigma) a_{2}\right)^{-2} \frac{1}{8}{ }_{2} F_{1}\left(2 q, 2,3, \frac{\left(a_{2}-a_{1}\right)(1-2 \varsigma)}{2\left(u a_{1}+(1-\varsigma) a_{2}\right)}\right), \\
& B_{3}^{\diamond}=\left(\varsigma a_{2}+(1-\varsigma) a_{1}\right)^{-2} \frac{1}{8}{ }_{2} F_{1}\left(2 q, 2,3, \frac{\left(a_{2}-a_{1}\right)(1-2 \varsigma)}{2\left(u b+(1-\varsigma) a_{1}\right)}\right), \\
& B_{4}^{\diamond}=\left(\varsigma a_{2}+(1-\varsigma) a_{1}\right)^{-2} \frac{1}{24}{ }_{2} F_{1}\left(2 q, 3,4, \frac{\left(a_{2}-a_{1}\right)(1-2 \varsigma)}{\left(u b+(1-\varsigma) a_{1}\right)}\right) .
\end{aligned}
$$

In order to establish our next refinement of Ostrowski's inequality, we use Hölder's inequality.

Theorem 3. Let $\Lambda: I \rightarrow \mathbb{R}$ be a differentiable mapping on $I^{\circ}$, where $a_{1}, a_{2} \in I$ with $a_{1}<a_{2}$. Let $q \geq 1$ and define $p$ by the equality $\frac{1}{p}+\frac{1}{q}=1$. If the function $\left|\Lambda^{\prime}\right|^{q}$ is harmonically convex, then, for $\varrho \in[0,1], x \in\left[a_{1}, a_{2}\right]$, and $\varsigma \in[0,1] \backslash\left\{\frac{1}{2}\right\}$, the following inequality is valid:

$$
\begin{aligned}
|T(\Lambda, \varrho, \zeta, x)| \leq & a_{1} a_{2}\left(a_{2}-a_{1}\right)(1-2 \varsigma)\left(\left(\psi_{1}^{*}\right)^{\frac{1}{p}}\left(\left|\Lambda^{\prime}\left(\frac{a_{1} a_{2}}{A_{a_{2}}}\right)\right|^{q} C_{1}^{*}+\left|\Lambda^{\prime}\left(\frac{a_{1} a_{2}}{A_{a_{1}}}\right)\right|^{q} C_{2}^{*}\right)^{\frac{1}{q}}\right. \\
& \left.+\left(\psi_{2}^{*}\right)^{\frac{1}{p}}\left(\left|\Lambda^{\prime}\left(\frac{a_{1} a_{2}}{A_{a_{2}}}\right)\right|^{q} C_{3}^{*}+\left|\Lambda^{\prime}\left(\frac{a_{1} a_{2}}{A_{a_{1}}}\right)\right|^{q} C_{4}^{*}\right)^{\frac{1}{q}}\right)
\end{aligned}
$$

where

$$
\begin{aligned}
& \psi_{1}^{*}=\int_{0}^{\frac{a_{1}\left(a_{2}-x\right)}{x\left(a_{2}-a_{1}\right)}} \frac{1}{A_{\tau}^{2 p}} \mathrm{~d} \tau \\
& =\left(\varsigma a_{1}+(1-\varsigma) a_{2}\right)^{-2 p} \frac{a_{1}\left(a_{2}-x\right)}{x\left(a_{2}-a_{1}\right)}{ }_{2} F_{1}\left(2 q, 1,2,\left(\frac{a_{1}\left(a_{2}-x\right)(1-2 \varsigma)}{x\left(u a_{1}+(1-\varsigma) a_{2}\right)}\right)\right), \\
& \psi_{2}^{*}=\int_{\frac{a_{1}\left(a_{2}-x\right)}{x\left(a_{2}-a_{1}\right)}}^{1} \frac{1}{A_{\tau}^{2 p}} \mathrm{~d} \tau \\
& =\left(\varsigma a_{1}+(1-\varsigma) a_{2}\right)^{-2 p}\left({ }_{2} F_{1}\left(2 q, 1,2,\left(\frac{\left(a_{2}-a_{1}\right)(1-2 \varsigma)}{\left(u a_{1}+(1-\varsigma) a_{2}\right)}\right)\right)\right. \\
& \left.-\frac{a_{1}\left(a_{2}-x\right)}{x\left(a_{2}-a_{1}\right)}{ }_{2} F_{1}\left(2 q, 1,2,\left(\frac{a_{1}\left(a_{2}-x\right)(1-2 \zeta)}{x\left(u a_{1}+(1-\varsigma) a_{2}\right)}\right)\right)\right), \\
& C_{1}^{*}=\int_{0}^{\frac{a_{1}\left(a_{2}-x\right)}{x\left(a_{2}-a_{1}\right)}} \tau\left|\tau-\varrho \frac{a_{1}\left(a_{2}-x\right)}{x\left(a_{2}-a_{1}\right)}\right|^{q} \mathrm{~d} \tau \\
& =\frac{1}{(q+1)(q+2)}\left(\frac{a_{1}\left(a_{2}-x\right)}{x\left(a_{2}-a_{1}\right)}\right)^{q+2}\left(\varrho^{q+2}-(1-\varrho)^{q+2}+(1-\varrho)^{q+1}(q+2)\right),
\end{aligned}
$$

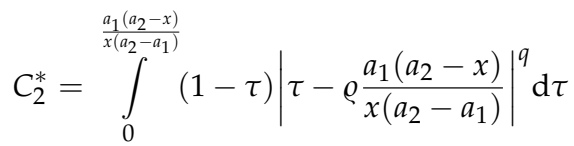

$$
\begin{aligned}
& =\frac{1}{(q+1)(q+2)}\left(\frac{a_{1}\left(a_{2}-x\right)}{x\left(a_{2}-a_{1}\right)}\right)^{q+1} \\
& \times\left\{\left(\left(\frac{a_{1}\left(a_{2}-x\right)}{x\left(a_{2}-a_{1}\right)}\right)\left((1-\varrho)^{q+2}-\varrho^{q+2}-(q+2)(1-\varrho)^{q+1}\right)\right)+(q+2)\left(\varrho^{q+1}+(1-\varrho)^{q+1}\right)\right\},
\end{aligned}
$$




$$
\begin{aligned}
C_{3}^{*}= & \int_{\frac{a_{1}\left(a_{2}-x\right)}{x x\left(a_{2}-a_{1}\right)}}^{1} \tau\left|\tau-1+\varrho \frac{a_{2}\left(x-a_{1}\right)}{x\left(a_{2}-a_{1}\right)}\right|^{q} \mathrm{~d} \tau \\
= & \frac{1}{(q+1)(q+2)}\left(\frac{a_{2}\left(x-a_{1}\right)}{x\left(a_{2}-a_{1}\right)}\right)^{q+1} \\
& \times\left\{\left(\left(\frac{a_{2}\left(x-a_{1}\right)}{x\left(a_{2}-a_{1}\right)}\right)\left((1-\varrho)^{q+2}-\varrho^{q+2}-(q+2)(1-\varrho)^{q+1}\right)\right)+(q+2)\left(\varrho^{q+1}+(1-\varrho)^{q+1}\right)\right\}, \\
C_{4}^{*}= & \int_{\frac{a_{1}\left(a_{2}-x\right)}{x\left(a_{2}-a_{1}\right)}}^{1}(1-\tau)\left|\tau-1+\varrho \frac{a_{2}\left(x-a_{1}\right)}{x\left(a_{2}-a_{1}\right)}\right|^{q} \mathrm{~d} \tau \\
= & \frac{1}{(q+1)(q+2)}\left(\frac{a_{2}\left(x-a_{1}\right)}{x\left(a_{2}-a_{1}\right)}\right)^{q+2}\left(\varrho^{q+2}-(1-\varrho)^{q+2}+(1-\varrho)^{q+1}(q+2)\right) .
\end{aligned}
$$

Proof. For brevity, we denote $A_{a_{1}}=\varsigma a_{1}+(1-\varsigma) a_{2}$ and $A_{a_{2}}=\varsigma a_{2}+(1-\varsigma) a_{1}$. Taking modulus in Lemma 1 and using Hölder's inequality, we have

$$
\begin{aligned}
& |T(\Lambda, \varrho, \varsigma, x)| \\
& \leq\left\{\int_{0}^{\frac{a_{1}\left(a_{2}-x\right)}{x\left(a_{2}-a_{1}\right)}}\left|\tau-\varrho \frac{a_{1}\left(a_{2}-x\right)}{x\left(a_{2}-a_{1}\right)} \Lambda^{\prime}\left(\frac{a_{1} a_{2}}{A_{\tau}}\right) \frac{a_{1} a_{2}\left(a_{2}-a_{1}\right)(1-2 \zeta)}{A_{\tau}^{2}}\right| \mathrm{d} \tau\right. \\
& \left.+\int_{\frac{a_{1}\left(a_{2}-x\right)}{x\left(a_{2}-a_{1}\right)}}^{1}\left|\tau-1+\varrho \frac{a_{2}\left(x-a_{1}\right)}{x\left(a_{2}-a_{1}\right)} \Lambda^{\prime}\left(\frac{a_{1} a_{2}}{A_{\tau}}\right) \frac{a_{1} a_{2}\left(a_{2}-a_{1}\right)(1-2 \zeta)}{A_{\tau}^{2}}\right| \mathrm{d} \tau\right\} \\
& \leq a_{1} a_{2}\left(a_{2}-a_{1}\right)(1-2 \varsigma)\left\{\left(\int_{0}^{\frac{a_{1}\left(a_{2}-x\right)}{x\left(a_{2}-a_{1}\right)}} \frac{1}{\left(A_{\tau}\right)^{2 p}} \mathrm{~d} \tau\right)^{\frac{1}{p}}\left(\int_{0}^{\frac{a_{1}\left(a_{2}-x\right)}{x\left(a_{2}-a_{1}\right)}}\left|\tau-\varrho \frac{a_{1}\left(a_{2}-x\right)}{x\left(a_{2}-a_{1}\right)}\right|^{q}\left|\Lambda^{\prime}\left(\frac{a_{1} a_{2}}{A_{\tau}}\right)\right|^{q} \mathrm{~d} \tau\right)^{\frac{1}{q}}\right. \\
& \left.+\left(\int_{\frac{a_{1}\left(a_{2}-x\right)}{x\left(a_{2}-a_{1}\right)}}^{1} \frac{1}{\left(A_{\tau}\right)^{2 p}} \mathrm{~d} \tau\right)^{\frac{1}{p}}\left(\int_{\frac{a_{1}\left(a_{2}-x\right)}{x\left(a_{2}-a_{1}\right)}}^{1}\left|\tau-1+\varrho \frac{a_{2}\left(x-a_{1}\right)}{x\left(a_{2}-a_{1}\right)}\right|^{q}\left|\Lambda^{\prime}\left(\frac{a_{1} a_{2}}{A_{\tau}}\right)\right|^{q} \mathrm{~d} \tau\right)^{\frac{1}{q}}\right\} \\
& =a_{1} a_{2}\left(a_{2}-a_{1}\right)(1-2 \varsigma) K_{3} .
\end{aligned}
$$


Using the harmonic convexity of $\left|\Lambda^{\prime}\right|^{q}$, we get

$$
\begin{aligned}
K_{3} \leq & \left\{( \int _ { 0 } ^ { \frac { a _ { 1 } ( a _ { 2 } - x ) } { x ( a _ { 2 } - a _ { 1 } ) } } \frac { 1 } { ( A _ { \tau } ) ^ { 2 p } } \mathrm { d } \tau ) ^ { \frac { 1 } { p } } \left(\left|\Lambda^{\prime}\left(\frac{a_{1} a_{2}}{A_{a_{2}}}\right)\right|_{0}^{q} \int_{0}^{\frac{a_{1}\left(a_{2}-x\right)}{x\left(a_{2}-a_{1}\right)}} \tau\left|\tau-\varrho \frac{a_{1}\left(a_{2}-x\right)}{x\left(a_{2}-a_{1}\right)}\right|^{q} \mathrm{~d} \tau\right.\right. \\
& \left.+\left|\Lambda^{\prime}\left(\frac{a_{1} a_{2}}{A_{a_{1}}}\right)\right|^{q} \int_{0}^{\frac{a_{1}\left(a_{2}-x\right)}{x\left(a_{2}-a_{1}\right)}}(1-\tau)\left|\tau-\varrho \frac{a_{1}\left(a_{2}-x\right)}{x\left(a_{2}-a_{1}\right)}\right|^{q} \mathrm{~d} \tau\right)^{\frac{1}{q}} \\
& \left.+\mid \int_{\frac{a_{1}\left(a_{2}-x\right)}{x\left(a_{2}-a_{1}\right)}}^{\frac{1}{\left(A_{\tau}\right.}} \frac{1}{\left(A_{\tau}\right)^{2 p}} \mathrm{~d} \tau\right)^{\frac{1}{p}}\left(\left|\Lambda^{\prime}\left(\frac{a_{1} a_{2}}{A_{a_{2}}}\right)\right|^{q} \int_{\frac{a_{1}\left(a_{2}-x\right)}{x\left(a_{2}-a_{1}\right)}}^{q}\left|\tau-1+\varrho \frac{a_{2}\left(x-a_{1}\right)}{x\left(a_{2}-a_{1}\right)}\right|^{q} \mathrm{~d} \tau\right. \\
& \left.\left.+\left|\Lambda^{\prime}\left(\frac{a_{1} a_{2}}{A_{a_{1}}}\right)\right|_{\frac{a_{1}\left(a_{2}-x\right)}{x\left(a_{2}-a_{1}\right)}}^{q}(1-\tau)\left|\tau-1+\varrho \frac{a_{2}\left(x-a_{1}\right)}{x\left(a_{2}-a_{1}\right)}\right|^{q} \mathrm{~d} \tau\right)^{\frac{1}{q}}\right\} \\
= & \left(\psi_{1}^{*}\right)^{\frac{1}{p}}\left(\left|\Lambda^{\prime}\left(\frac{a_{1} a_{2}}{A_{a_{2}}}\right)\right|^{q} C_{1}^{*}+\left|\Lambda^{\prime}\left(\frac{a_{1} a_{2}}{A_{a_{1}}}\right)\right|^{q} C_{2}^{*}\right)^{\frac{1}{q}} \\
& \left.+\left(\psi_{2}^{*}\right)^{\frac{1}{p}}\left(\left|\Lambda^{\prime}\left(\frac{a_{1} a_{2}}{A_{a_{2}}}\right)\right|^{q} C_{3}^{*}+\left|\Lambda^{\prime}\left(\frac{a_{1} a_{2}}{A_{a_{1}}}\right)\right|^{q} C_{4}^{*}\right)^{\frac{1}{q}}\right)
\end{aligned}
$$

This completes the proof.

Corollary 4. Under the assumptions of Theorem 3, if we choose $\varsigma=0$ in 5 , then

$$
\begin{aligned}
|T(\Lambda, \varrho, 0, x)| \leq & a_{1} a_{2}\left(a_{2}-a_{1}\right)\left(\left(\psi_{1}^{* *}\right)^{\frac{1}{p}}\left(\left|\Lambda^{\prime}\left(\frac{a_{1} a_{2}}{A_{a_{2}}}\right)\right|^{q} C_{1}^{*}+\left|\Lambda^{\prime}\left(\frac{a_{1} a_{2}}{A_{a_{1}}}\right)\right|^{q} C_{2}^{*}\right)^{\frac{1}{q}}\right. \\
& \left.+\left(\psi_{2}^{* *}\right)^{\frac{1}{p}}\left(\left|\Lambda^{\prime}\left(\frac{a_{1} a_{2}}{A_{a_{2}}}\right)\right|^{q} C_{3}^{*}+\left|\Lambda^{\prime}\left(\frac{a_{1} a_{2}}{A_{a_{1}}}\right)\right|^{q} C_{4}^{*}\right)^{\frac{1}{q}}\right),
\end{aligned}
$$

where

$$
\begin{aligned}
& \psi_{1}^{* *}=a_{2}{ }^{-2 p} \frac{a_{1}\left(a_{2}-x\right)}{x\left(a_{2}-a_{1}\right)}{ }_{2} F_{1}\left(2 q, 1,2,\left(\frac{a_{1}\left(a_{2}-x\right)}{x b}\right)\right), \\
& \psi_{2}^{* *}=a_{2}{ }^{-2 p}\left({ }_{2} F_{1}\left(2 q, 1,2,\left(\frac{\left(a_{2}-a_{1}\right)}{a_{2}}\right)\right)-\frac{a_{1}\left(a_{2}-x\right)}{x\left(a_{2}-a_{1}\right)}{ }_{2} F_{1}\left(2 q, 1,2,\left(\frac{a_{1}\left(a_{2}-x\right)}{x b}\right)\right)\right),
\end{aligned}
$$

and where $C_{1}^{*}, C_{2}^{*}, C_{3}^{*}$ and $C_{4}^{*}$ are given in Theorem 3 . 
Corollary 5. Under the assumptions of Theorem 3, if we choose $\varrho=0$ in (5), then

$$
\begin{aligned}
|T(\Lambda, 0, \zeta, x)| \leq & a_{1} a_{2}\left(a_{2}-a_{1}\right)(1-2 \zeta)\left(\left(\psi_{1}^{*}\right)^{\frac{1}{p}}\left(\left|\Lambda^{\prime}\left(\frac{a_{1} a_{2}}{A_{a_{2}}}\right)\right|^{q} C_{1}^{* *}+\left|\Lambda^{\prime}\left(\frac{a_{1} a_{2}}{A_{a_{1}}}\right)\right|^{q} C_{2}^{* *}\right)^{\frac{1}{q}}\right. \\
& \left.+\left(\psi_{2}^{*}\right)^{\frac{1}{p}}\left(\left|\Lambda^{\prime}\left(\frac{a_{1} a_{2}}{A_{a_{2}}}\right)\right|^{q} C_{3}^{* *}+\left|\Lambda^{\prime}\left(\frac{a_{1} a_{2}}{A_{a_{1}}}\right)\right|^{q} C_{4}^{* *}\right)^{\frac{1}{q}}\right),
\end{aligned}
$$

where $\psi_{1}^{*}, \psi_{2}^{*}$ are given in Theorem 3 and

$$
\begin{aligned}
& C_{1}^{* *}=\int_{0}^{\frac{a_{1}\left(a_{2}-x\right)}{x\left(a_{2}-a_{1}\right)}} \tau^{q+1} \mathrm{~d} \tau=\frac{1}{(q+2)}\left(\frac{a_{1}\left(a_{2}-x\right)}{x\left(a_{2}-a_{1}\right)}\right)^{q+2}, \\
& C_{2}^{* *}=\int_{0}^{\frac{a_{1}\left(a_{2}-x\right)}{x\left(a_{2}-a_{1}\right)}}(1-\tau) \tau^{q} \mathrm{~d} \tau \\
& =\frac{1}{(q+1)(q+2)}\left(\frac{a_{1}\left(a_{2}-x\right)}{x\left(a_{2}-a_{1}\right)}\right)^{q+1}\left\{\left(\left(\frac{a_{1}\left(a_{2}-x\right)}{x\left(a_{2}-a_{1}\right)}\right)(-q-1)\right)+(q+2)\right\}, \\
& C_{3}^{* *}=\int_{\frac{a_{1}\left(a_{2}-x\right)}{x\left(a_{2}-a_{1}\right)}}^{1} \tau|\tau-1|^{q} \mathrm{~d} \tau \\
& =\frac{1}{(q+1)(q+2)}\left(\frac{a_{2}\left(x-a_{1}\right)}{x\left(a_{2}-a_{1}\right)}\right)^{q+1}\left\{\left(\left(\frac{a_{2}\left(x-a_{1}\right)}{x\left(a_{2}-a_{1}\right)}\right)(-q-1)\right)+(q+2)\right\}, \\
& C_{4}^{* *}=\int_{\frac{a_{1}\left(a_{2}-x\right)}{x\left(a_{2}-a_{1}\right)}}^{1}(1-\tau)|\tau-1|^{q} \mathrm{~d} \tau=\frac{1}{(q+2)}\left(\frac{a_{2}\left(x-a_{1}\right)}{x\left(a_{2}-a_{1}\right)}\right)^{q+2} \text {. }
\end{aligned}
$$

Corollary 6. Under the assumptions of Theorem 3, if we choose $\varrho=0$ and $x=\frac{2 a_{1} a_{2}}{a_{1}+a_{2}}$ in Corollary 5, then

$$
\begin{aligned}
\left|T\left(\Lambda, 0, \varsigma, \frac{2 a_{1} a_{2}}{a_{1}+a_{2}}\right)\right| \leq & a_{1} a_{2}\left(a_{2}-a_{1}\right)(1-2 \varsigma)\left(\left(\psi_{1}^{* *}\right)^{\frac{1}{p}}\left(\left|\Lambda^{\prime}\left(\frac{a_{1} a_{2}}{A_{a_{2}}}\right)\right|^{q} C_{1}^{* * *}+\left|\Lambda^{\prime}\left(\frac{a_{1} a_{2}}{A_{a_{1}}}\right)\right|^{q} C_{2}^{* * *}\right)^{\frac{1}{q}}\right. \\
& \left.+\left(\psi_{2}^{* *}\right)^{\frac{1}{p}}\left(\left|\Lambda^{\prime}\left(\frac{a_{1} a_{2}}{A_{a_{2}}}\right)\right|^{q} C_{3}^{* * *}+\left|\Lambda^{\prime}\left(\frac{a_{1} a_{2}}{A_{a_{1}}}\right)\right|^{q} C_{4}^{* * *}\right)^{\frac{1}{q}}\right),
\end{aligned}
$$


where

$$
\begin{aligned}
& \psi_{1}^{* *}=\int_{0}^{\frac{1}{2}} \frac{1}{A_{\tau}^{2 p}} \mathrm{~d} \tau \\
& =\left(\varsigma a_{1}+(1-\varsigma) a_{2}\right)^{-2 p} \frac{1}{2}{ }_{2} F_{1}\left(2 q, 1,2,\left(\frac{\left(a_{2}-a_{1}\right)(1-2 \varsigma)}{2\left(u a_{1}+(1-\varsigma) a_{2}\right)}\right)\right) \text {, } \\
& \psi_{2}^{* *}=\int_{\frac{1}{2}}^{1} \frac{1}{A_{\tau}^{2 p}} \mathrm{~d} \tau \\
& =\left(s a_{1}+(1-\varsigma) a_{2}\right)^{-2 p}\left({ }_{2} F_{1}\left(2 q, 1,2,\left(\frac{\left(a_{2}-a_{1}\right)(1-2 \varsigma)}{\left(u a_{1}+(1-\varsigma) a_{2}\right)}\right)\right)\right. \\
& \left.-\frac{1}{2}{ }_{2} F_{1}\left(2 q, 1,2,\left(\frac{\left(a_{2}-a_{1}\right)(1-2 \varsigma)}{2\left(u a_{1}+(1-\varsigma) a_{2}\right)}\right)\right)\right) \text {, } \\
& C_{1}^{* * *}=\int_{0}^{\frac{1}{2}} \tau^{q+1} \mathrm{~d} \tau=\frac{1}{(q+2)}\left(\frac{1}{2}\right)^{q+2} \\
& C_{2}^{* * *}=\int_{0}^{\frac{1}{2}}(1-\tau) \tau^{q} \mathrm{~d} \tau=\frac{q+3}{2(q+1)(q+2)}\left(\frac{1}{2}\right)^{q+1} \\
& C_{3}^{* * *}=\int_{\frac{a_{1}}{a_{1}+a_{2}}}^{1} \tau|\tau-1|^{q} \mathrm{~d} \tau=\frac{q+3}{2(q+1)(q+2)}\left(\frac{1}{2}\right)^{q+1} \\
& C_{4}^{* * *}=\int_{\frac{a_{1}}{a_{1}+a_{2}}}^{1}(1-\tau)|\tau-1|^{q} \mathrm{~d} \tau=\frac{1}{(q+2)}\left(\frac{1}{2}\right)^{q+2}
\end{aligned}
$$

Theorem 4. Let the notions and hypothesis be as in Theorem 3. Then, the following inequality is true for $\varrho \in[0,1], x \in\left[a_{1}, a_{2}\right]$ and $\varsigma \in[0,1] \backslash\left\{\frac{1}{2}\right\}$ :

$$
\begin{aligned}
|T(\Lambda, \varrho, \zeta, x)| \leq & a_{1} a_{2}\left(a_{2}-a_{1}\right)(1-2 \varsigma)\left(\left(\psi_{1}\right)^{\frac{1}{p}}\left(\left|\Lambda^{\prime}\left(\frac{a_{1} a_{2}}{A_{a_{2}}}\right)\right|^{q} C_{1}+\left|\Lambda^{\prime}\left(\frac{a_{1} a_{2}}{A_{a_{1}}}\right)\right|^{q} C_{2}\right)^{\frac{1}{q}}\right. \\
& \left.+\left(\psi_{2}\right)^{\frac{1}{p}}\left(\left|\Lambda^{\prime}\left(\frac{a_{1} a_{2}}{A_{a_{2}}}\right)\right|^{q} C_{3}+\left|\Lambda^{\prime}\left(\frac{a_{1} a_{2}}{A_{a_{1}}}\right)\right|^{q} C_{4}\right)^{\frac{1}{q}}\right)
\end{aligned}
$$

where

$$
\begin{aligned}
& \psi_{1}=\int_{0}^{\frac{a_{1}\left(a_{2}-x\right)}{x\left(a_{2}-a_{1}\right)}}\left|\tau-\varrho \frac{a_{1}\left(a_{2}-x\right)}{x\left(a_{2}-a_{1}\right)}\right|^{p} \mathrm{~d} \tau=\left(\frac{\varrho^{p+1}+(1-\varrho)^{p+1}}{p+1}\right)\left(\frac{a_{1}\left(a_{2}-x\right)}{x\left(a_{2}-a_{1}\right)}\right)^{p+1}, \\
& \psi_{2}=\int_{\frac{a_{1}\left(a_{2}-x\right)}{x\left(a_{2}-a_{1}\right)}}^{1}\left|\tau-1+\varrho \frac{a_{2}\left(x-a_{1}\right)}{x\left(a_{2}-a_{1}\right)}\right|^{p} \mathrm{~d} \tau=\left(\frac{\varrho^{p+1}+(1-\varrho)^{p+1}}{p+1}\right)\left(\frac{a_{2}\left(x-a_{1}\right)}{x\left(a_{2}-a_{1}\right)}\right)^{p+1},
\end{aligned}
$$




$$
\begin{aligned}
& C_{1}=\int_{0}^{\frac{a_{1}\left(a_{2}-x\right)}{x\left(a_{2}-a_{1}\right)}} \tau\left[\tau\left(\varsigma a_{2}+(1-\varsigma) a_{1}\right)+(1-\tau)\left(\varsigma a_{1}+(1-\varsigma) a_{2}\right)\right]^{-2 q} \mathrm{~d} \tau \\
& =\frac{\left(\varsigma a_{1}+(1-\varsigma) a_{2}\right)^{-2 q}}{2}\left(\frac{a_{1}\left(a_{2}-x\right)}{x\left(a_{2}-a_{1}\right)}\right)^{2}{ }_{2} F_{1}\left(2 q, 2,3,\left(\frac{a_{1}\left(a_{2}-x\right)(1-2 \varsigma)}{x\left(u a_{1}+(1-\varsigma) a_{2}\right)}\right)\right), \\
& \frac{a_{1}\left(a_{2}-x\right)}{x\left(a_{2}-a_{1}\right)} \\
& C_{2}=\int_{0}(1-\tau)\left[\tau\left(\varsigma a_{2}+(1-\varsigma) a_{1}\right)+(1-\tau)\left(\varsigma a_{1}+(1-\varsigma) a_{2}\right)\right]^{-2 q} \mathrm{~d} \tau \\
& =\left(\varsigma a_{1}+(1-\varsigma) a_{2}\right)^{-2 q}\left(\frac{a_{1}\left(a_{2}-x\right)}{x\left(a_{2}-a_{1}\right)}\right)\left({ }_{2} F_{1}\left(2 q, 1,2,\left(\frac{a_{1}\left(a_{2}-x\right)(1-2 \varsigma)}{x\left(u a_{1}+(1-\varsigma) a_{2}\right)}\right)\right)\right. \\
& \left.-\left(\frac{a_{1}\left(a_{2}-x\right)}{2 x\left(a_{2}-a_{1}\right)}\right){ }_{2} F_{1}\left(2 q, 2,3,\left(\frac{a_{1}\left(a_{2}-x\right)(1-2 \varsigma)}{x\left(u a_{1}+(1-\varsigma) a_{2}\right)}\right)\right)\right), \\
& C_{3}=\int_{\frac{a_{1}\left(a_{2}-x\right)}{x\left(a_{2}-a_{1}\right)}}^{1} \tau\left[\tau\left(\varsigma a_{2}+(1-\varsigma) a_{1}\right)+(1-\tau)\left(\varsigma a_{1}+(1-\varsigma) a_{2}\right)\right]^{-2 q} \mathrm{~d} \tau \\
& =\frac{\left(\varsigma a_{1}+(1-\varsigma) a_{2}\right)^{-2 q}}{2}\left({ }_{2} F_{1}\left(2 q, 2,3,\left(\frac{(1-2 \varsigma)\left(a_{2}-x\right)}{\left(u a_{1}+(1-\varsigma) a_{2}\right)}\right)\right)\right. \\
& \left.-\left(\frac{a_{1}\left(a_{2}-x\right)}{x\left(a_{2}-a_{1}\right)}\right)^{2}{ }_{2} F_{1}\left(2 q, 2,3,\left(\frac{a_{1}\left(a_{2}-x\right)(1-2 \varsigma)}{x\left(u a_{1}+(1-\varsigma) a_{2}\right)}\right)\right)\right) \\
& C_{4}=\int_{\frac{a_{1}\left(a_{2}-x\right)}{x\left(a_{2}-a_{1}\right)}}^{1}(1-\tau)\left[\tau\left(\varsigma a_{2}+(1-\varsigma) a_{1}\right)+(1-\tau)\left(\varsigma a_{1}+(1-\varsigma) a_{2}\right)\right]^{-2 q} \mathrm{~d} \tau \\
& =\left(\varsigma a_{1}+(1-\varsigma) a_{2}\right)^{-2 q}\left(\frac{1}{2}{ }_{2} F_{1}\left(2 q, 1,3,\left(\frac{(1-2 \varsigma)\left(a_{2}-x\right)}{\left(u a_{1}+(1-\varsigma) a_{2}\right)}\right)\right)\right. \\
& -\left(\frac{a_{1}\left(a_{2}-x\right)}{x\left(a_{2}-a_{1}\right)}\right){ }_{2} F_{1}\left(2 q, 1,2,\left(\frac{a_{1}\left(a_{2}-x\right)(1-2 \varsigma)}{x\left(u a_{1}+(1-\varsigma) a_{2}\right)}\right)\right) \\
& +\frac{1}{2}\left(\frac{a_{1}\left(a_{2}-x\right)}{x\left(a_{2}-a_{1}\right)}\right)^{2}{ }_{2} F_{1}\left(2 q, 2,3,\left(\frac{a_{1}\left(a_{2}-x\right)(1-2 \varsigma)}{x\left(u a_{1}+(1-\varsigma) a_{2}\right)}\right)\right)
\end{aligned}
$$

Proof. For simplicity, we assume that $A_{a_{1}}=\varsigma a_{1}+(1-\varsigma) a_{2}$ and $A_{a_{2}}=\varsigma a_{2}+(1-\varsigma) a_{1}$. Taking modulus in Lemma 1 and using Hölder's inequality, we have

$$
\begin{aligned}
|T(\Lambda, \varrho, \zeta, x)| \leq & \left\{\int_{0}^{\frac{a_{1}\left(a_{2}-x\right)}{x\left(a_{2}-a_{1}\right)}}\left|\tau-\varrho \frac{a_{1}\left(a_{2}-x\right)}{x\left(a_{2}-a_{1}\right)}\right|\left|\Lambda^{\prime}\left(\frac{a_{1} a_{2}}{A_{\tau}}\right) \frac{a_{1} a_{2}\left(a_{2}-a_{1}\right)(1-2 \zeta)}{A_{\tau}^{2}}\right| \mathrm{d} \tau\right. \\
& \left.+\int_{\frac{a_{1}\left(a_{2}-x\right)}{x\left(a_{2}-a_{1}\right)}}^{1}\left|\tau-1+\varrho \frac{a_{2}\left(x-a_{1}\right)}{x\left(a_{2}-a_{1}\right)}\right|\left|\Lambda^{\prime}\left(\frac{a_{1} a_{2}}{A_{\tau}}\right) \frac{a_{1} a_{2}\left(a_{2}-a_{1}\right)(1-2 \zeta)}{A_{\tau}^{2}}\right| \mathrm{d} \tau\right\}
\end{aligned}
$$




$$
\begin{aligned}
\leq & a_{1} a_{2}\left(a_{2}-a_{1}\right)(1-2 \varsigma)\left\{\left(\int_{0}^{\frac{a_{1}\left(a_{2}-x\right)}{x\left(a_{2}-a_{1}\right)}}\left|\tau-\varrho \frac{a_{1}\left(a_{2}-x\right)}{x\left(a_{2}-a_{1}\right)}\right|^{p} \mathrm{~d} \tau\right)^{\frac{1}{p}}\left(\int_{0}^{\frac{a_{1}\left(a_{2}-x\right)}{x}\left(a_{2}-a_{1}\right)}\left|\Lambda^{\prime}\left(\frac{a_{1} a_{2}}{A_{\tau}}\right) \frac{1}{A_{\tau}^{2}}\right|^{q} \mathrm{~d} \tau\right)^{\frac{1}{q}}\right. \\
& \left.+\left(\int_{\frac{a_{1}\left(a_{2}-x\right)}{x\left(a_{2}-a_{1}\right)}}^{1}\left|\tau-1+\varrho \frac{a_{2}\left(x-a_{1}\right)}{x\left(a_{2}-a_{1}\right)}\right|^{p} \mathrm{~d} \tau\right)^{\frac{1}{p}}\left(\int_{\frac{a_{1}\left(a_{2}-x\right)}{x\left(a_{2}-a_{1}\right)}}^{1}\left|\Lambda^{\prime}\left(\frac{a_{1} a_{2}}{A_{\tau}}\right) \frac{1}{A_{\tau}^{2}}\right|^{q} \mathrm{~d} \tau\right)^{\frac{1}{q}}\right\} \\
= & a_{1} a_{2}\left(a_{2}-a_{1}\right)(1-2 \varsigma) K_{4} .
\end{aligned}
$$

Using the harmonic convexity of $\left|\Lambda^{\prime}\right|^{q}$, we get

$$
\begin{aligned}
K_{4} \leq & \left\{\left(\int_{0}^{\frac{a_{1}\left(a_{2}-x\right)}{x\left(a_{2}-a_{1}\right)}}\left|\tau-\varrho \frac{a_{1}\left(a_{2}-x\right)}{x\left(a_{2}-a_{1}\right)}\right|^{p} \mathrm{~d} \tau\right)^{\frac{1}{p}}\right. \\
& \times\left(\left|\Lambda^{\prime}\left(\frac{a_{1} a_{2}}{A_{a_{2}}}\right)\right|_{0}^{q} \int_{0}^{\frac{a_{1}\left(a_{2}-x\right)}{x\left(a_{2}-a_{1}\right)}} \frac{\tau}{\left(A_{\tau}\right)^{2 q}} \mathrm{~d} \tau+\left|\Lambda^{\prime}\left(\frac{a_{1} a_{2}}{A_{a_{1}}}\right)\right|^{q} \int_{0}^{\frac{a_{1}\left(a_{2}-x\right)}{x\left(a_{2}-a_{1}\right)}} \frac{(1-\tau)}{\left(A_{\tau}\right)^{2 q}} \mathrm{~d} \tau\right)^{\frac{1}{q}} \\
& +\left(\int_{\frac{a_{1}\left(a_{2}-x\right)}{x\left(a_{2}-a_{1}\right)}}^{1}\left|\tau-1+\varrho \frac{a_{2}\left(x-a_{1}\right)}{x\left(a_{2}-a_{1}\right)}\right|^{p} \mathrm{~d} \tau||^{\frac{1}{p}}\right. \\
& \left.\times\left(\left|\Lambda^{\prime}\left(\frac{a_{1} a_{2}}{A_{a_{2}}}\right)\right|^{q} \int_{\frac{a_{1}\left(a_{2}-x\right)}{x\left(a_{2}-a_{1}\right)}}^{1} \frac{\tau}{\left(A_{\tau}\right)^{2 q}} \mathrm{~d} \tau+\left|\Lambda^{\prime}\left(\frac{a_{1} a_{2}}{A_{a_{1}}}\right)\right|^{q} \int_{\frac{a_{1}\left(a_{2}-x\right)}{x\left(a_{2}-a_{1}\right)}}^{1} \frac{(1-\tau)}{\left(A_{\tau}\right)^{2 q}} \mathrm{~d} \tau\right)^{\frac{1}{q}}\right\} \\
= & \left(\left(\psi_{1}\right)^{\frac{1}{p}}\left(\left|\Lambda^{\prime}\left(\frac{a_{1} a_{2}}{A_{a_{2}}}\right)\right|^{q} C_{1}+\left|\Lambda^{\prime}\left(\frac{a_{1} a_{2}}{A_{a_{1}}}\right)\right|^{q} C_{2}\right)^{\frac{1}{q}}\right. \\
& \left.+\left(\psi_{2}\right)^{\frac{1}{p}}\left(\left|\Lambda^{\prime}\left(\frac{a_{1} a_{2}}{A_{a_{2}}}\right)\right|^{q} C_{3}+\left|\Lambda^{\prime}\left(\frac{a_{1} a_{2}}{A_{a_{1}}}\right)\right|^{q} C_{4}\right)^{\frac{1}{q}}\right) .
\end{aligned}
$$

This completes the proof.

Corollary 7. Under the assumptions of Theorem 4, if we choose $\varsigma=0$ in (6), then

$$
\begin{aligned}
|T(\Lambda, \varrho, 0, x)| \leq & a_{1} a_{2}\left(a_{2}-a_{1}\right)(1-2 \varsigma)\left(\left(\psi_{1}\right)^{\frac{1}{p}}\left(\left|\Lambda^{\prime}\left(\frac{a_{1} a_{2}}{A_{a_{2}}}\right)\right|^{q} C_{1}^{\diamond}+\left|\Lambda^{\prime}\left(\frac{a_{1} a_{2}}{A_{a_{1}}}\right)\right|^{q} C_{2}^{\diamond}\right)^{\frac{1}{q}}\right. \\
& \left.+\left(\psi_{2}\right)^{\frac{1}{p}}\left(\left|\Lambda^{\prime}\left(\frac{a_{1} a_{2}}{A_{a_{2}}}\right)\right|^{q} C_{3}^{\diamond}+\left|\Lambda^{\prime}\left(\frac{a_{1} a_{2}}{A_{a_{1}}}\right)\right|^{q} C_{4}^{\diamond}\right)^{\frac{1}{q}}\right)
\end{aligned}
$$


where $\psi_{1}, \psi_{2}$ are given in Theorem 4 and

$$
\begin{aligned}
C_{1}^{\diamond}= & \int_{0}^{\frac{a_{1}\left(a_{2}-x\right)}{x\left(a_{2}-a_{1}\right)}} \tau\left[\tau a_{1}+(1-\tau) a_{2}\right]^{-2 q} \mathrm{~d} \tau \\
= & \frac{a_{2}-2 q}{2}\left(\frac{a_{1}\left(a_{2}-x\right)}{x\left(a_{2}-a_{1}\right)}\right)^{2}{ }_{2} F_{1}\left(2 q, 2,3, \frac{a_{1}\left(a_{2}-x\right)}{x b}\right), \\
C_{2}^{\diamond}= & \int_{0}^{\frac{a_{1}\left(a_{2}-x\right)}{\left.x x a_{2}-a_{1}\right)}}(1-\tau)\left[\tau a_{1}+(1-\tau) a_{2}\right]^{-2 q} \mathrm{~d} \tau \\
= & a_{2}{ }^{-2 q}\left(\frac{a_{1}\left(a_{2}-x\right)}{x\left(a_{2}-a_{1}\right)}\right)\left({ }_{2} F_{1}\left(2 q, 1,2, \frac{a_{1}\left(a_{2}-x\right)}{x b}\right)-\left(\frac{a_{1}\left(a_{2}-x\right)}{2 x\left(a_{2}-a_{1}\right)}\right){ }_{2} F_{1}\left(2 q, 2,3, \frac{a_{1}\left(a_{2}-x\right)}{x b}\right)\right), \\
C_{3}^{\diamond}= & \int_{\frac{a_{1}\left(a_{2}-x\right)}{x\left(a_{2}-a_{1}\right)}}^{1} \tau\left[\tau a_{1}+(1-\tau) a_{2}\right]^{-2 q} \mathrm{~d} \tau \\
= & \frac{a_{2}-2 q}{2}\left({ }_{2} F_{1}\left(2 q, 2,3, \frac{\left(a_{2}-x\right)}{a_{2}}\right)-\left(\frac{a_{1}\left(a_{2}-x\right)}{x\left(a_{2}-a_{1}\right)}\right)^{2}{ }_{2} F_{1}\left(2 q, 2,3, \frac{a_{1}\left(a_{2}-x\right)}{x b}\right)\right), \\
C_{4}^{\diamond}= & \int_{\frac{a_{1}\left(a_{2}-x\right)}{x\left(a_{2}-a_{1}\right)}}^{1}(1-\tau)\left[\tau a_{1}+(1-\tau) a_{2}\right]^{-2 q} \mathrm{~d} \tau \\
= & a_{2}{ }^{-2 q}\left(\frac{1}{2}{ }_{2} F_{1}\left(2 q, 1,3, \frac{\left(a_{2}-x\right)}{a_{2}}\right)\right. \\
& -\left(\frac{a_{1}\left(a_{2}-x\right)}{x\left(a_{2}-a_{1}\right)}\right){ }_{2} F_{1}\left(2 q, 1,2,\left(\frac{a_{1}\left(a_{2}-x\right)}{x b}\right)+\frac{1}{2}\left(\frac{a_{1}\left(a_{2}-x\right)}{x\left(a_{2}-a_{1}\right)}\right)^{2}{ }_{2} F_{1}\left(2 q, 2,3, \frac{a_{1}\left(a_{2}-x\right)}{x b}\right)\right) .
\end{aligned}
$$

Corollary 8. Under the assumptions of Theorem 4, if we choose $Q=0$ in (6), then

$$
\begin{aligned}
|T(\Lambda, 0, \varsigma, x)| \leq & a_{1} a_{2}\left(a_{2}-a_{1}\right)(1-2 \varsigma)\left(\left(\psi_{1}^{\diamond}\right)^{\frac{1}{p}}\left(\left|\Lambda^{\prime}\left(\frac{a_{1} a_{2}}{A_{a_{2}}}\right)\right|^{q} C_{1}+\left|\Lambda^{\prime}\left(\frac{a_{1} a_{2}}{A_{a_{1}}}\right)\right|^{q} C_{2}\right)^{\frac{1}{q}}\right. \\
& \left.+\left(\psi_{2}^{\diamond}\right)^{\frac{1}{p}}\left(\left|\Lambda^{\prime}\left(\frac{a_{1} a_{2}}{A_{a_{2}}}\right)\right|^{q} C_{3}+\left|\Lambda^{\prime}\left(\frac{a_{1} a_{2}}{A_{a_{1}}}\right)\right|^{q} C_{4}\right)^{\frac{1}{q}}\right)
\end{aligned}
$$

where

$$
\begin{aligned}
& \psi_{1}^{\diamond}=\int_{0}^{\frac{a_{1}\left(a_{2}-x\right)}{x\left(a_{2}-a_{1}\right)}}|\tau|^{p} \mathrm{~d} \tau=\left(\frac{1}{p+1}\right)\left(\frac{a_{1}\left(a_{2}-x\right)}{x\left(a_{2}-a_{1}\right)}\right)^{p+1}, \\
& \psi_{2}^{\diamond}=\int_{\frac{a_{1}\left(a_{2}-x\right)}{x\left(a_{2}-a_{1}\right)}}^{1}|\tau-1|^{p} \mathrm{~d} \tau=\left(\frac{1}{p+1}\right)\left(\frac{a_{2}\left(x-a_{1}\right)}{x\left(a_{2}-a_{1}\right)}\right)^{p+1},
\end{aligned}
$$

where $C_{1}, C_{2}, C_{3}$ and $C_{4}$ are given in Theorem 4. 
Corollary 9. Under the assumptions of Theorem 4 , if we choose $\varsigma=0$ and $x=\frac{2 a_{1} a_{2}}{a_{1}+a_{2}}$ in Corollary 8 , then

$$
\begin{aligned}
\left|T\left(\Lambda, 0,0, \frac{2 a_{1} a_{2}}{a_{1}+a_{2}}\right)\right| \leq & a_{1} a_{2}\left(a_{2}-a_{1}\right)(1-2 \zeta)\left(\left(\psi_{1}^{\diamond \diamond}\right)^{\frac{1}{p}}\left(\left|\Lambda^{\prime}\left(a_{2}\right)\right|^{q} C_{1}^{\diamond \diamond}+\left|\Lambda^{\prime}\left(a_{1}\right)\right|^{q} C_{2}^{\diamond \diamond}\right)^{\frac{1}{q}}\right. \\
& \left.+\left(\psi_{2}^{\diamond \diamond}\right)^{\frac{1}{p}}\left(\left|\Lambda^{\prime}\left(a_{2}\right)\right|^{q} C_{3}^{\diamond \diamond}+\left|\Lambda^{\prime}\left(a_{1}\right)\right|^{q} C_{4}^{\diamond \diamond}\right)^{\frac{1}{q}}\right),
\end{aligned}
$$

where

$$
\begin{aligned}
& \psi_{1}^{\diamond \diamond}=\int_{0}^{\frac{a_{1}}{a_{1}+a_{2}}}|\tau|^{p} \mathrm{~d} \tau=\left(\frac{1}{p+1}\right)\left(\frac{1}{2}\right)^{p+1},
\end{aligned}
$$

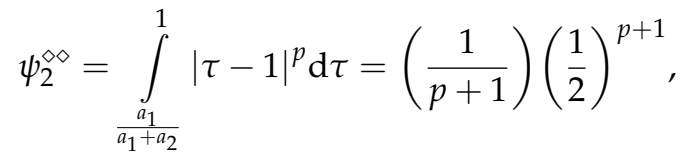

$$
\begin{aligned}
& C_{1}^{\diamond \diamond}=\frac{1}{\left(a_{2}-a_{1}\right)^{2}}\left[\frac{a_{2}^{2-2 q}}{1-2 q}-\frac{a_{2}\left(\frac{a_{1}+a_{2}}{2}\right)^{1-2 q}}{1-2 q}+\frac{\left(\frac{a_{1}+a_{2}}{2}\right)^{2-2 q}}{2-2 q}-\frac{a_{2}^{2-2 q}}{2-2 q}\right], \\
& C_{2}^{\diamond \diamond}=\frac{1}{\left(a_{2}-a_{1}\right)^{2}}\left[\frac{a_{2}^{2-2 q}}{2-2 q}+\frac{a_{1}\left(\frac{a_{1}+a_{2}}{2}\right)^{1-2 q}}{1-2 q}-\frac{\left(\frac{a_{1}+a_{2}}{2}\right)^{2-2 q}}{2-2 q}-\frac{a_{1} a_{2}{ }^{1-2 q}}{1-2 q}\right] \text {, } \\
& C_{3}^{\diamond \diamond}=\frac{1}{\left(a_{2}-a_{1}\right)^{2}}\left[\frac{a_{1}^{2-2 q}}{2-2 q}+\frac{a_{2}\left(\frac{a_{1}+a_{2}}{2}\right)^{1-2 q}}{1-2 q}-\frac{\left(\frac{a_{1}+a_{2}}{2}\right)^{2-2 q}}{2-2 q}-\frac{a_{1}{ }^{1-2 q} a_{2}}{1-2 q}\right] \text {, } \\
& C_{4}^{\diamond \diamond}=\frac{1}{\left(a_{2}-a_{1}\right)^{2}}\left[\frac{a_{1}^{2-2 q}}{1-2 q}-\frac{a_{1}\left(\frac{a_{1}+a_{2}}{2}\right)^{1-2 q}}{1-2 q}+\frac{\left(\frac{a_{1}+a_{2}}{2}\right)^{2-2 q}}{2-2 q}-\frac{a_{1}^{2-2 q}}{2-2 q}\right] .
\end{aligned}
$$

\section{Applications}

In this section, we present some applications to special means. We recall the following well known special means for two nonnegative real numbers:

1. The Arithmetic Mean: $A\left(a_{1}, a_{2}\right):=\frac{a_{1}+a_{2}}{2}$

2. The Geometric Mean: $G\left(a_{1}, a_{2}\right):=\sqrt{a_{1} a_{2}}$

3. The Harmonic Mean: $H\left(a_{1}, a_{2}\right):=\frac{2 a_{1} a_{2}}{a_{1}+a_{2}}$

4. The Logarithmic Mean: $L\left(a_{1}, a_{2}\right):=\frac{a_{2}-a_{1}}{\ln a_{2}-\ln a_{1}}$

5. The $p$-Logarithmic mean: $L_{p}\left(a_{1}, a_{2}\right):=\left(\frac{a_{2} p+1-a_{1} p+1}{(p+1)\left(a_{2}-a_{1}\right)}\right)^{\frac{1}{p}}, p \in \mathbb{R} \backslash\{-1,0\}$.

We now discuss our results. 
Proposition 1. For $0<a_{1}<a_{2}$, we have

$$
\begin{aligned}
& \left|H\left(a_{1}, a_{2}\right)-\frac{G^{2}\left(a_{1}, a_{2}\right)}{L\left(a_{1}, a_{2}\right)}\right| \\
& \leq a_{1} a_{2}\left(a_{2}-a_{1}\right)\left[\left(\psi_{1}^{\diamond \diamond}\right)^{\frac{1}{p}}\left(\frac{1}{\left(a_{2}-a_{1}\right)^{2}}\left(\frac{a_{2}^{2-2 q}}{2-2 q}-\frac{a_{1} a_{2}{ }^{1-2 q}}{1-2 q}-\frac{\left(a_{2}-a_{1}\right) A^{1-2 q}\left(a_{1}, a_{2}\right)}{1-2 q}\right)\right)^{\frac{1}{q}}\right. \\
& \left.+\left(\psi_{2}^{\diamond \diamond}\right)^{\frac{1}{p}}\left(\frac{1}{\left(a_{2}-a_{1}\right)^{2}}\left(\frac{a_{1}^{2-2 q}}{2-2 q}-\frac{a_{1}^{1-2 q} a_{2}}{1-2 q}+\frac{\left(a_{2}-a_{1}\right) A^{1-2 q}\left(a_{1}, a_{2}\right)}{1-2 q}\right)\right)^{\frac{1}{q}}\right] .
\end{aligned}
$$

Proof. The assertion follows from the Corollary 9 , for $\Lambda(x)=x$.

Proposition 2. For $0<a_{1}<a_{2}$, we have

$$
\begin{aligned}
& \left|H^{p+2}\left(a_{1}, a_{2}\right)-G^{2}\left(a_{1}, a_{2}\right) L_{p}^{p}\left(a_{1}, a_{2}\right)\right| \\
& \leq a_{1} a_{2}\left(a_{2}-a_{1}\right)\left[( \psi _ { 1 } ^ { \diamond } ) ^ { \frac { 1 } { p } } \left(\frac{(p+2) a_{2}{ }^{p+1}}{\left(a_{2}-a_{1}\right)^{2}}\left(\frac{a_{2}^{2-2 q}}{1-2 q}-\frac{b A^{1-2 q}\left(a_{1}, a_{2}\right)}{1-2 q}\right)+\frac{(p+2) a_{1}{ }^{p+1}}{\left(a_{2}-a_{1}\right)^{2}}\left(\frac{a_{1} a_{2}{ }^{1-2 q}}{1-2 q}\right.\right.\right. \\
& \left.\left.-\frac{a A^{1-2 q}\left(a_{1}, a_{2}\right)}{1-2 q}\right)+\frac{(p+1)(p+2) A^{2-2 q}\left(a_{1}, a_{2}\right)}{\left(a_{2}-a_{1}\right)(2-2 q)} L_{p}^{p}\left(a_{1}, a_{2}\right)-\frac{(p+1)(p+2) a_{2}^{2-2 q}}{\left(a_{2}-a_{1}\right)(2-2 q)} L_{p}^{p}\left(a_{1}, a_{2}\right)\right)^{\frac{1}{q}} \\
& +\left(\psi_{2}^{\diamond \diamond}\right)^{\frac{1}{p}}\left(\frac{(p+2) a_{2}{ }^{p+1}}{\left(a_{2}-a_{1}\right)^{2}}\left(-\frac{a_{1}^{1-2 q} a_{2}}{1-2 q}+\frac{b A^{1-2 q}\left(a_{1}, a_{2}\right)}{1-2 q}\right)+\frac{(p+2) a_{1}{ }^{p+1}}{\left(a_{2}-a_{1}\right)^{2}}\left(\frac{a_{1}^{2-2 q}}{1-2 q}\right.\right. \\
& \left.\left.\left.\left.-\frac{a A^{1-2 q}\left(a_{1}, a_{2}\right)}{1-2 q}\right)-\frac{(p+1)(p+2) A^{2-2 q}\left(a_{1}, a_{2}\right)}{\left(a_{2}-a_{1}\right)(2-2 q)} L_{p}^{p}\left(a_{1}, a_{2}\right)+\frac{(p+1)(p+2) a_{1}{ }^{2-2 q}}{\left(a_{2}-a_{1}\right)(2-2 q)} L_{p}^{p}\left(a_{1}, a_{2}\right)\right)^{\frac{1}{q}}\right)^{\frac{1}{q}}\right] .
\end{aligned}
$$

Proof. The assertion follows from the Corollary 9 , for $\Lambda(x)=x^{p+2}$.

\section{Conclusions}

We have derived several new refinements of Ostrowski type of integral inequalities using the class of harmonically convex functions. We have discussed several new special cases of the obtained results as well. This shows that our results are quite unifying. In order to show the significance of the main results, we have also presented some applications of our main results to special means. Recently, authors [15,16] have obtained HermiteHadamard's inequality on higher dimensions. In addition, it will be an interesting problem for future research to consider the results obtained in this paper on higher dimensions.

Author Contributions: Writing—original draft, N.A., M.U.A., M.Z.J., M.T.R., M.V.M., M.A.N. and K.I.N. All authors have read and agreed to the published version of the manuscript.

Funding: This research received no external funding.

Acknowledgments: The authors are thankful to the editor as well as to the anonymous referees for their valuable comments and suggestions that helped improve the presentation of this paper.

Conflicts of Interest: The authors declare no conflict of interest.

\section{References}

1. İşcan, İ. Ostrowski type inequalities for harmonically s-convex functions. Konuralp J. Math. 2015, 3, 63-74.

2. Al-Azemi, F.; Calin, O. Asian options with harmonic average. Appl. Math. Inf. Sci. 2015, 9, 1-9.

3. Noor, M.A. Advanced Convex and Numerical Analysis, Lecture Notes; COMSATS Institute of Information Technology: Islamabad, Pakistan, 2018.

4. İşcan, İ. Hermite-Hadamard type inequalities for harmonically convex functions. Hacettepe J. Math. Stat. 2014, 43, 935-942. [CrossRef]

5. Alomari, M.; Darus, M.; Dragomir, S.S.; Cerone, P. Ostrowski type inequalities for functions whose derivatives are s-convex in the second sense. Appl. Math. Lett. 2010, 23, 1071-1076. [CrossRef] 
6. Ardic, M.A.; Akdemir, A.O.; Set, E. New Ostrowski like inequalities for GG-convex and GA-convex functions. Math. Ineq. Appl. 2016, 19, 1159-1168.

7. Budak, H.; Sarikaya, M.Z. On generalized Ostrowski-type inequalities for functions whose first derivatives absolute values are convex. Turk. J. Math. 2016, 40, 1193-1210. [CrossRef]

8. Budak, H.; Sarikaya, M.Z. On weighted Ostrowski type inequalities for functions of two variables with bounded variation. J. Nonlinear Funct. Anal. 2017, 2017, 23.

9. Khurshid, Y.; Khan, M.A.; Chu, Y.M. Ostrowski type inequalities involving conformable integrals via preinvex functions. AIP Adv. 2020, 10, 055204. [CrossRef]

10. Koroglu, T. New Ostrowski type inequalities for harmonically convex functions. New Trends Math. Sci. 2018. [CrossRef]

11. Mohsen, B.-B.; Awan, M.U.; Noor, M.A.; Mihai, M.V.; Noor, K.I. New Ostrowski like inequalities involving the functions having harmonic $h$-convexity property and applications. J. Math. Inequal. 2019, 13, 621-644. [CrossRef]

12. Set, E. Some new generalizations of Ostrowski type inequalities for $s$-convex functions via fractional integral operators. Filomat 2018, 32, 5595-5609. [CrossRef]

13. Sun, W. Local fractional Ostrowski type inequalities involving generalized $h$-convex functions and some applications for generalized moments. Fractals 2020. [CrossRef]

14. Dragomir, S.S.; Rassias, T.M. Ostrowski Type Inequalities and Applications in Numerical Integration; Springer: Dordrecht, The Netherlands, 2002.

15. Lu, J.-F.; Steinerberger, S. A dimension-free Hermite-Hadamard inequality via gradient estimates for the torsion function. Proc. Amer. Math. Soc. 2020, 148, 673-679. [CrossRef]

16. Steinerberger, S. The Hermite-Hadamard inequality in higher dimensions. J. Geom. Anal. 2020, 30, 466-483. [CrossRef] 